\title{
Optical phonons in carbon nanotubes: Kohn anomalies, Peierls distortions, and dynamic effects
}

\author{
Stefano Piscanec, ${ }^{1}$ Michele Lazzeri, ${ }^{2}$ J. Robertson, ${ }^{2}$ Andrea C. Ferrari, ${ }^{1}, *$ and Francesco Mauri ${ }^{2, \dagger}$ \\ ${ }^{1}$ Engineering Department, Cambridge University, 9 JJ Thomson Avenue, Cambridge CB3 OFA, United Kingdom \\ ${ }^{2}$ Institut de Minéralogie et de Physique des Milieux Condensés, Université Paris VI, 4 Place Jussieu, Paris, France
}

(Received 23 September 2006; published 31 January 2007)

\begin{abstract}
We present a detailed study of the vibrational properties of single wall carbon nanotubes (SWNTs). The phonon dispersions of SWNTs are strongly shaped by the effects of electron-phonon coupling. We analyze the separate contributions of curvature and confinement. Confinement plays a major role in modifying SWNT phonons and is often more relevant than curvature. Due to their one-dimensional character, metallic tubes are expected to undergo Peierls distortions (PD) at $T=0 \mathrm{~K}$. At finite temperature, PD are no longer present, but phonons with atomic displacements similar to those of the PD are affected by strong Kohn anomalies (KA). We investigate by density functional theory (DFT) KA and PD in metallic SWNTs with diameters up to $3 \mathrm{~nm}$, in the electronic temperature range from $4 \mathrm{~K}$ to $3000 \mathrm{~K}$. We then derive a set of simple formulas accounting for all the DFT results. Finally, we prove that the static approach, commonly used for the evaluation of phonon frequencies in solids, fails because of the SWNTs reduced dimensionality. The correct description of KA in metallic SWNTs can be obtained only by using a dynamical approach, beyond the adiabatic Born-Oppenheimer approximation, by taking into account nonadiabatic contributions. Dynamic effects induce significant changes in the occurrence and shape of Kohn anomalies. We show that the SWNT Raman $G$ peak can only be interpreted considering the combined dynamic, curvature and confinement effects. We assign the $G^{+}$and $G^{-}$ peaks of metallic SWNTs to TO (circumferential) and LO (axial) modes, respectively, the opposite of semiconducting SWNTs.
\end{abstract}

DOI: 10.1103/PhysRevB.75.035427 PACS number(s): 78.67.Ch, 63.20.Dj, 63.20.Kr, 71.15.Mb

\section{INTRODUCTION}

Carbon nanotubes are at the center of nanotechnology research. ${ }^{1,2}$ The determination of their structure, phonon dispersions, and Raman spectra is a most intense area of investigation since their discovery. ${ }^{3-6}$ Single wall nanotubes (SWNTs) can be described either as giant molecules or onedimensional crystals. Due to their reduced dimensionality, confinement effects play a fundamental role in shaping their physical properties, such as the metallic or semiconducting character or the electron transport mechanisms. Optical phonons of SWNTs are extremely important. Indeed, they contribute the most intense features in the Raman spectra. These are commonly used to sort metallic from semiconducting SWNTs. Electron scattering by optical phonons also sets the ultimate limit of high field ballistic transport, ${ }^{7-10}$ due to hot phonon generation. ${ }^{10,11}$ Electron-phonon coupling (EPC) is the key physical parameter necessary to quantify the phonons interaction with electrons. In metallic SWNTs the EPC strongly affects the phonon frequencies, giving rise to Kohn anomalies (KA) $)^{12-16}$ and Peierls distortions (PD). ${ }^{15,17-24}$ A correct understanding and a quantitatively precise description of electron-phonon coupling, Kohn anomalies, and Peierls distortions in SWNTs is then of prime scientific and technological interest.

Experimentally, the most commonly studied SWNTs have diameters from $0.8 \mathrm{~nm}$, up to $3 \mathrm{~nm}$, with hundreds to thousands of atoms per unit cell. Covering such diameter range pushes the limits of computational approaches. SWNTs optical phonons were calculated using phonon zone folding, ${ }^{18,25-27}$ tight binding ${ }^{28-31}$ (TB), density functional theory (DFT), ${ }^{17-20,27,32,33}$ and symmetry-adapted models. ${ }^{31,34-37}$ The simplest approach to get SWNT phonons, consists in folding those of graphene $e^{4,5,18,38}$ This requires the prior calculation of graphene phonons either by $\mathrm{TB}, 25,26,38$ DFT ${ }^{12,18,27,39}$ or by other approaches. ${ }^{37}$ Phonon zone folding (PZF) is not limited by the size of the SWNT unit cell. However, even if SWNT electrons are well described by folded graphene, ${ }^{40}$ this is not always true for phonons. We have shown that graphene has two KA at $\boldsymbol{\Gamma}$ and $\mathbf{K} .{ }^{12}$ Due to their reduced dimensionality, metallic SWNTs have much stronger KA than graphene. ${ }^{12-14}$ Thus, folded graphene cannot give a reliable phonon dispersion of metallic SWNTs close to the Kohn anomalies, no matter how accurate the graphene phonons. ${ }^{12,39}$ On the other hand, semiconducting SWNTs cannot have KA. So, even in this case, folded graphene may not correctly describe the phonons.

These problems can be overcome by means of TB or DFT calculations. Indeed, the EPC effects can be taken into account in TB models. ${ }^{8,41,42}$ However, the EPC strongly depend on the TB parametrization, and contrasting values are found in literature. ${ }^{8,10,41-43}$ Also, most TB works failed to identify KA. ${ }^{28-30}$ DFT directly includes EPC effects, and, being an $a b$ initio technique, does not rely on external adjustable parameters. However, this better accuracy is obtained at the price of a significant increase of the computational time demand, which makes the evaluation of SWNT properties a challenge. As a result, most DFT works focus on extremely small tubes ${ }^{19,20}$ and/or achiral tubes. ${ }^{17,18,27}$. Finally, in all the approaches used to compute phonon frequencies, the time dependent nature of phonons is neglected. This approximation, which results in a static description of the atomic vibration, is usually legitimate in tridimensional crystals, ${ }^{44,45}$ but, as we will show, does not necessarily hold for onedimensional systems.

In this paper we show that SWNTs are a major exception, and that a correct description of their optical phonons can 
only be achieved by carefully considering the dynamical nature of phonons. We first analyze the static case and single out the effects of curvature and confinement. Confinement plays a major role in shaping SWNT phonons and is often more relevant than curvature. We present an electronic zone folding method allowing the static DFT calculation of confinement effects on phonon dispersions and EPC of SWNTs of any diameter and chirality, and for any electronic temperature $\left(T_{e}\right)$. We investigate KA and PD in metallic SWNTs with diameters up to $3 \mathrm{~nm}$ and for $T_{e}=4$ to $3000 \mathrm{~K}$. We present a simple analytic model exactly accounting for all the static DFT results. Finally, we prove that dynamic effects induce significant changes in KA occurrence and shape. We show that the SWNT Raman $G$ peak can only be interpreted considering the combined dynamic, curvature and confinement effects. We assign the $G^{+}$and $G^{-}$peaks of metallic SWNTs to TO (circumferential) and LO (axial) modes, the opposite of semiconducting SWNTs. A family dependence of the $G$ peak position in semiconducting tubes is observed.

This paper is organized as follows. In Sec. II we introduce the background concepts and definitions. Section III presents our EPC calculations. Section IV and V present, respectively, the study of the effect of pure confinement within the static and the dynamic approach. Section VI highlights the influence of curvature on the SWNT phonons. Finally, Sec. VII assigns the $G^{+}$and $G^{-}$Raman peaks of SWNTs.

\section{BACKGROUND}

Before presenting our numerical and analytic results, it is necessary to introduce and discuss a set of background concepts and definitions, which will be used thorough the paper, in order to highlight the differences between our approach and others in literature.

\section{A. SWNTs unit cell and graphene zone folding}

SWNTs are usually identified by means of their chiral indices $n$ and $m .{ }^{4,5}$ The determination of the chiral indices is done by unrolling the unit cell of the tube and comparing it to an infinite graphene sheet. The unrolled unit cell is a rectangular sheet of graphene that can be described by means of the vectors $\mathbf{C}_{h}$ and $\mathbf{T}$. $\mathbf{C}_{h}$ is known as the chiral vector and has the same length as the circumference of the tube. $\mathbf{T}$ is known as the translation vector and defines the translational symmetry of the tube. The crystal lattice of graphene can be defined by two vectors, $\mathbf{a}_{1}$ and $\mathbf{a}_{2}$, forming a $\pi / 3$ angle. $\mathbf{C}_{h}$ and $\mathbf{T}$ can be projected on $\mathbf{a}_{1}$ and $\mathbf{a}_{2},{ }^{4,5}$

$$
\mathbf{C}_{h}=n \mathbf{a}_{1}+m \mathbf{a}_{2}, \quad \mathbf{T}=\frac{(2 m+n) \mathbf{a}_{1}}{d_{R}}-\frac{(2 n+m) \mathbf{a}_{2}}{d_{R}},
$$

where $n, m$ are defined as the chiral indices and $d_{R}$ $=\operatorname{gcd}(2 n+m, 2 m+n)(\operatorname{gcd}$ represents greatest common divisor).

Wave vectors in nanotubes must be commensurate to the tube circumference. They can be represented on the unrolled unit cell of the tube, and are in the form

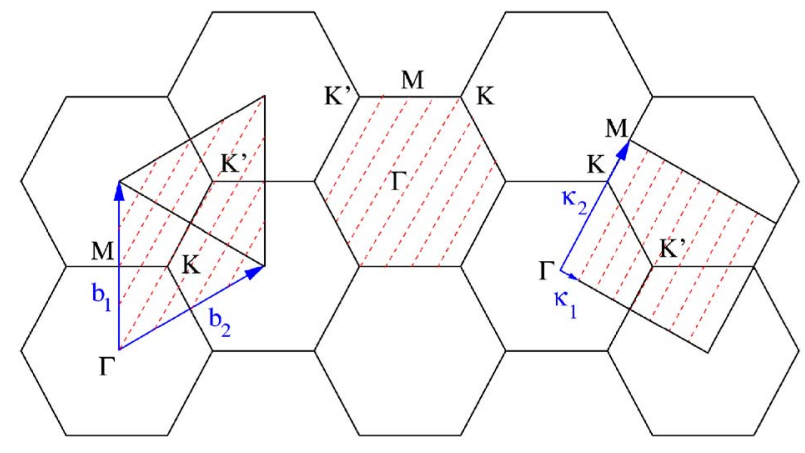

FIG. 1. (Color online) Three equivalent representations of the zone-folding lines for a $(4,4)$ tube, $\mathrm{ZF}_{(4,4)}$, in the reciprocal space of graphene. Left, plot of $\mathrm{ZF}_{(4,4)}$ in the hcp BZ of graphene; the primitive vectors $\mathbf{b}_{1}$ and $\mathbf{b}_{2}$ are plotted in blue, the lines of $Z F_{(4,4)}$ are plotted in red. Center, plot of $\mathrm{ZF}_{(4,4)}$ in the hexagonal representation of the graphene $\mathrm{BZ}$. On the right-hand side, plot of $\mathrm{ZF}_{(4,4)}$ emphasizing the vectors $\mathbf{K}_{1}$ and $\mathbf{K}_{2}$. The length of $\mathbf{K}_{2}$ corresponds to the length of the $(4,4)$ tube BZ.

$$
\mathbf{k}=\mathbf{k}_{\|}+\mathbf{k}_{\perp}=\mu \mathbf{K}_{1}+\nu \mathbf{K}_{2},
$$

where $\mathbf{K}_{2}$ is parallel to $\mathbf{T}$, its modulus $K_{2}=2 \pi / T$ correspond to the length of the tube's Brillouin zone (BZ), and $\nu$ can assume any real value; $\mathbf{K}_{1}$ is perpendicular to $\mathbf{T}$, and $\mu \mathbf{K}_{1}$ must be commensurate to the SWNT circumference. Thus $K_{1}=2 \pi / C_{h}, \mu \in \mathbb{N}$, and $\mu \in\left[N_{c} / 2-1, N_{c}-1\right], N_{c}$ being the number of unit cells of graphene contained in the unrolled unit cell of the tube. The number of nodes of a wave function along the tube circumference is given by $i=2 \mu$., $4,46,47$

Figure 1 plots the wave vectors commensurate to a $(4,4)$ tube on the reciprocal space of graphene, showing that they are located on lines oriented along $\mathbf{K}_{2}$, spaced by $\mathbf{K}_{1}$ and including the $\boldsymbol{\Gamma}$ point. ${ }^{4,5,46,47}$ For the generic $(n, m)$ tube, we refer to this ensemble of cutting lines as $\mathrm{ZF}_{(n, m)}$. The procedure of using wave vectors of graphene to describe wave vectors of a SWNT is known as zone folding (ZF). Using the correspondence set by ZF between graphene and tube wave vectors, it is possible to use the electrons and phonons of graphene to obtain the band structure or the phonon dispersions of SWNTs. ${ }^{4,5,46,47}$

For clarity, in the rest of this paper we will distinguish the electron and phonon wave vectors by labeling them, respectively, with the symbols $\mathbf{k}$ and $\mathbf{q}$.

\section{B. LO-TO phonons in graphene, graphite, and nanotubes}

The analysis of the SWNT Raman $G$ band shows that the phonons deriving from the $\boldsymbol{\Gamma}-E_{2 g}$ mode of graphene, of $A$ symmetry in SWNTs, provide the dominant contribution to the $G^{+}$and $G^{-}$Raman peaks. ${ }^{48,49}$ Thus, in this paper we focus on these modes and their phonon branches.

In literature, these phonons are often labeled using different criteria: ${ }^{4,5,18,50-52}$ (i) their symmetry, ${ }^{4,5,50}$ (ii) the direction of the atomic displacements with respect to the SWNT axis, radius or circumference, ${ }^{18}$ (iii) the longitudinal or transversal character of the graphene phonon branch from which they originate, ${ }^{51}$ (iiii) the longitudinal or transversal character relative to the SWNT. ${ }^{52}$ 
In this paper the following definition is used: a mode is longitudinal if $\boldsymbol{\epsilon} \| \mathbf{q}$ and transverse if $\boldsymbol{\epsilon} \perp \mathbf{q}$; where $\boldsymbol{\epsilon}$ is the phonon polarization (i.e., the direction of the atomic displacements) and $\mathbf{q}$ its wave vector, which also defines the direction of the phonon propagation.

In SWNTs, phonons can propagate only along the axis. As a result, modes with atomic displacements parallel to the axis are longitudinal, and those with atomic displacements perpendicular to the axis (i.e., along the circumference) are transversal.

In achiral SWNTs, symmetry forces the displacements of modes originating from graphene $\boldsymbol{\Gamma}-E_{2 g}$ to be either parallel or perpendicular to the axis. ${ }^{4,5}$ Thus, in armchair and zigzag SWNTs these are exactly LO and TO.

In chiral SWNTs, phonon polarizations are expected to be influenced by chiral angle and $\mathrm{C}-\mathrm{C}$ bonds orientation. ${ }^{51}$ Thus, the modes originating from the graphene $\Gamma-E_{2 g}$ should have only a prevalently LO or a prevalently TO character. Indeed, Ref. 51 proposed the LO-TO classification to be meaningless in chiral SWNTs. However, in Sec. VI we will show that for any SWNTs with diameter $>0.8 \mathrm{~nm}$, these modes are almost exactly LO and TO. We thus consider the LO-TO classification meaningful and convenient, also because it avoids different labeling in chiral and achiral SWNTs.

\section{Kohn anomalies and Peierls distortions}

In a metal, for certain phonons with a wave vector connecting two points of the Fermi surface it is possible to have an abrupt change of the electronic screening of the atomic vibrations. This results in a sudden softening of the phonon frequencies, which is called Kohn anomaly. ${ }^{53,54}$ In metallic SWNTs the Fermi surface consists of two points only. Thus, KA can occur only for phonons with $q=0$ or $q$ connecting the two Fermi points (Fig. 2). In graphene, a necessary condition for KA is a significant nonzero EPC (between electrons near the Fermi energy) for phonons at $\mathbf{q}=\boldsymbol{\Gamma}$ or $\mathbf{q}=\mathbf{K} .^{12}$ Due to their reduced dimensionality, metallic SWNTs are expected to have stronger KA than graphite and graphene, ${ }^{12-15,19,20}$ for the corresponding phonons.

One-dimensional (1D) metallic systems are predicted to be unstable at $T=0 \mathrm{~K} .{ }^{55} \mathrm{In}$ fact, in 1D metals it is always possible to find a lattice distortion that (i) opens a gap in the electronic structure, and (ii) such that the energy gained by gap opening compensates the strain induced by the deformation. This is called Peierls distortion. ${ }^{55}$ The possible presence of PD in SWNTs was argued as soon as their metallic behavior was predicted. ${ }^{46,47}$ Their geometry was investigated by several authors with contrasting results. It was proposed that they follow the atomic displacement patterns of LA phonons, ${ }^{21,22}$ of radial breathing modes (RBM), ${ }^{23}$ of solitwistons, ${ }^{24}$ or of optical phonons with $q=\Gamma$ or $q=2 k_{F},{ }^{13,15,17-20}$ where $k_{F}$ is the wave vector for which the electron gap is null. Note that the condition $q=2 k_{F}$ is equivalent to $q=K$ in Fig. 2.

For $T>0 \mathrm{~K}$, due to thermal excitation, states above the Fermi energy are populated. Thus, increasing the temperature reduces the energy gained by gap opening. PD are then

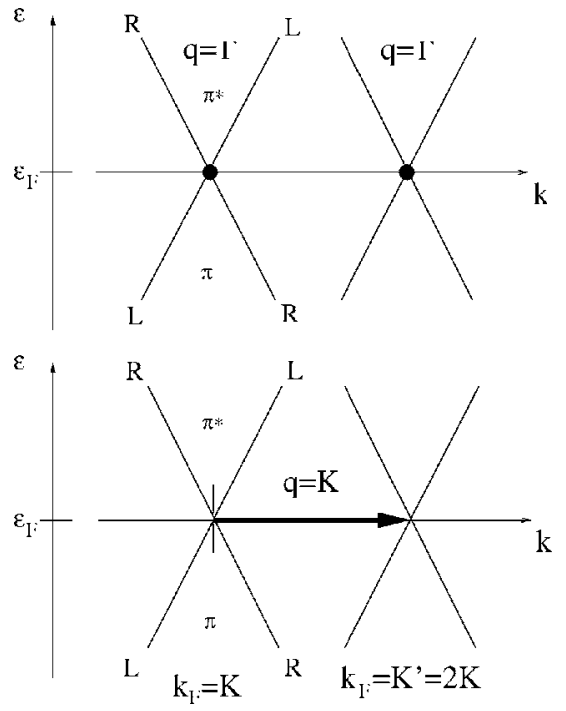

FIG. 2. Geometrical condition for the KA onset in metallic SWNTs: for $q=0$ a point of the Fermi surface is connected to itself. This corresponds to a KA at $\Gamma$. $q=K$ connects the two distinct Fermi points $\mathbf{K}$ and $\mathbf{K}^{\prime}$, giving a second KA. $\epsilon_{F}$ is the Fermi energy. We label the band with positive slope $L$, and that with negative slope $R$. In electron transport, this notation indicates bands where electrons enter the tube, respectively, from the left and right electrode.

stable only below a critical temperature $T_{\mathrm{PD}}$. This temperature is also known as the metal-semiconductor transition temperature, and its determination is crucial to understand the interplay between the possible onset of superconductivity and PD. ${ }^{15,19,20,56,57}$

For $T>T_{\mathrm{PD}}$, the energy gain achieved by gap opening is not sufficient to compensate the elastic strain, so the lattice will not undergo a permanent distortion. However, phonon modes having a pattern of atomic displacements corresponding to the PD can show an important softening. This effect is proportional to the energy gained by gap opening, and inversely proportional to the temperature. This mechanism, described by some authors as a Peierls-like mechanism, ${ }^{17,18}$ is, in fact, a particular case of Kohn anomaly. ${ }^{53}$

\section{Temperature effects}

Let us call $T_{e}$ and $T_{i}$ the electronic and the ionic temperature. $T_{i}$ corresponds to the energy associated to the atoms vibrations around their equilibrium positions. $T_{e}$ fixes the electronic states population close to the Fermi energy $\epsilon_{F}$,

$$
f_{\mathbf{k}, n}=\frac{1}{e^{\left(\epsilon_{\mathbf{k}, n}-\epsilon_{F}\right) / k_{B} T_{e}}+1},
$$

where $f_{\mathbf{k}, n}$ is the Fermi-Dirac occupation of the $n$th electronic band at $\mathbf{k}$, and $k_{B}$ is the Boltzmann's constant.

In metals, a finite electronic temperature always changes the electronic states occupation, while in materials with an electronic gap $E_{g}$, changes of the electronic occupancies are relevant only for $k_{B} T_{e}>E_{g}$.

The Peierls-distortion temperature $T_{\mathrm{PD}}$ depends only on the electronic structure of the system, and not on the lattice 
thermal energy. Thus, Peierls distortions are features of the phonon dispersions which directly depend on $T_{e}$. On the other hand, phonons can have a temperature dependence due to anharmonicity, ${ }^{54}$ which is determined by $T_{i}$.

It is thus essential to distinguish the different effects of $T_{e}$ and $T_{i}$ on the SWNT phonons. At thermal equilibrium, $T_{i}$ $=T_{e}=T_{S}$, where $T_{S}$ is the sample temperature. Thus, the correct phonon frequencies can be recovered only by taking simultaneously into account the contributions of Kohn anomalies and anharmonicity. The calculation of the anharmonic effects will be discussed elsewhere. ${ }^{58}$ To a first approximation, the anharmonic contribution is similar for phonons of similar energy. On the contrary, a key result of this paper will be to show that $T_{e}$ selectively acts only on the TO and LO phonon branches in metallic SWNTs, see Secs. IV and $\mathrm{V}$.

DFT, in its original formulation, is a ground state theory, thus $T_{e}=0 \mathrm{~K}$. DFT can be extended to include a finite real $T_{e}{ }^{59}$ This is done using the Mermin potential ${ }^{59}$ and populating the electronic states with the Fermi-Dirac distribution, Eq. (3). Because of the presence of KA and PD, the phonon frequencies of metallic SWNTs depend on $T_{e}$ and DFT calculation should always be done using the Mermin potential.

\section{E. The dynamical matrix}

We call $\Theta_{\mathbf{q}}$ (Ref. 60) the dynamical matrix projected on the phonons normal coordinates. Within time dependent perturbation theory, for a SWNT this is defined as (see, e.g., Eqs. 4.17a, 4.23 in Ref. 61):

$$
\begin{aligned}
\Theta_{q}= & \frac{2 T}{2 \pi} \sum_{m, n} \int_{\mathrm{BZ}} \frac{\left|D_{(k+q) n, k m}\right|^{2}\left(f_{k, m}-f_{k+q, n}\right)}{\epsilon_{k, m}-\epsilon_{k+q, n}+\hbar \omega_{q}+i \gamma} d k \\
& -\int \Delta n_{q}^{*}(\mathbf{r}) K\left(\mathbf{r}, \mathbf{r}^{\prime}\right) \Delta n_{q}\left(\mathbf{r}^{\prime}\right) d \mathbf{r} d \mathbf{r}^{\prime}+\int n(\mathbf{r}) \Delta^{2} V^{b}(\mathbf{r}) d \mathbf{r},
\end{aligned}
$$

where $2 \pi / T$ is the length of the tube BZ, $\Sigma_{m, n}$ is a sum on all the possible electronic transitions, $\int_{\mathrm{BZ}} d k$ is an integral over the one-dimensional tube BZ; $\epsilon_{k, n}$ is the energy of the electronic Bloch eigenstate with wave vector $k$ and band index $n$; $\gamma$ is a small real constant; $n(\mathbf{r})$ is the charge density; $K\left(\mathbf{r}, \mathbf{r}^{\prime}\right)=\delta^{2} E_{\mathrm{Hxc}}(n) / \delta n(\mathbf{r}) \delta n\left(\mathbf{r}^{\prime}\right)$, where $E_{\mathrm{Hxc}}(n)$ is the Hartree and exchange-correlation functional, and $\Delta^{2} V^{b}$ is the second derivative of the bare (purely ionic) potential; $D_{(k+q) n, k m}$ is the electron-phonon coupling matrix element,

$$
D_{(k+q) n, k m}=\left\langle k+q, n\left|\Delta V_{q}\left[\Delta n_{q}\right]\right| k, m\right\rangle,
$$

where $\Delta V_{q}$ and $\Delta n_{q}$ are the derivatives of Kohn-Sham potential and charge density with respect to displacement along the phonon normal coordinate; $|k, n\rangle$ is the electronic Bloch eigenstate of wave vector $k$ and band $n$. The phonon frequencies $\omega_{q}$ are derived from $\Theta_{q}$ as ${ }^{61}$

$$
\omega_{q}=\operatorname{Re}\left\{\sqrt{\frac{\Theta_{q}}{M}}\right\},
$$

where $M$ is the atomic mass of carbon.
It is important to note that Eq. (4) introduces a dependence of the dynamical matrix on the term $\hbar \omega_{q}+i \gamma$. This is a direct consequence of the perturbative approach used to derive Eq. (4), where the phonon is described as a timedependent perturbation of the system. ${ }^{61}$ This accounts for the dynamic nature of vibrations. In the rest of the paper, we will refer to the inclusion of the term $\hbar \omega_{q}+i \gamma$ in the expression of the dynamical matrix as "the inclusion of," or "the description of" dynamic effects (DE). This definition is introduced in opposition to the static approximation, which is described in Sec. II E 2.

\section{Dynamic effects}

Dynamic effects induce a correction in the denominators of terms proportional to the EPC in Eq. (4). In materials where the electronic gap is larger than the phonon energy, the dynamic effects are negligible. In metals, dynamic effects are negligible if the phonon energy is smaller than the electronic smearing for which the phonon calculations converge. In general, this is true for tridimensional metals. ${ }^{44,45}$ Thus, in this case the contribution of the dynamic effects to the dynamical matrix is negligible. That is why dynamic effects are not included in the most common ab initio codes for phonon calculations. ${ }^{68,69}$ However, we will show that this is not necessarily true for systems with reduced dimensionality. For example, as explained in the preceding section, onedimensional metallic systems undergo Peierls distortions, the phonon frequencies depend on the electronic temperature, and the phonon energy can be of the same order, or even larger than the electronic temperature. In this case, it is conceptually wrong to neglect the dynamic effects a priori.

Since metallic SWNTs can undergo Peierls distortions, the role of the dynamic effects on phonon frequency must be carefully considered and investigated. This will be done in Sec. V.

\section{The static approximation}

The Born-Oppenheimer, ${ }^{63}$ or adiabatic, or static approximation is equivalent to dropping the phonon energy $\hbar \omega_{q}$ in Eq. (4). ${ }^{64}$ Implementation of the static approximation results in what we call static DFPT. To the best of our knowledge, all existing DFPT codes implement static equations. Static DFPT gives the same results as other static approaches, such as frozen phonons, ${ }^{65}$ where phonons are obtained evaluating the energy associated to a static lattice distortion. The expression of $\Theta_{q}$ within the static approximation is obtained from Eq. (4) by setting to zero the phonon energy $\omega_{q}$ and the term $i \gamma$,

$$
\begin{aligned}
\Theta_{q}= & \frac{2 T}{2 \pi} \sum_{m, n} \int_{\mathrm{BZ}} \frac{f_{k, m}-f_{k+q, n}}{\epsilon_{k, m}-\epsilon_{k+q, n}}\left|D_{(k+q) n, k m}\right|^{2} d k \\
& -\int \Delta n_{q}^{*}(\mathbf{r}) K\left(\mathbf{r}, \mathbf{r}^{\prime}\right) \Delta n_{q}\left(\mathbf{r}^{\prime}\right) d \mathbf{r} d \mathbf{r}^{\prime}+\int n(\mathbf{r}) \Delta^{2} V^{b}(\mathbf{r}) d \mathbf{r} .
\end{aligned}
$$

Unlike in the dynamic case, within the static approximation $\Theta_{q}$ is real. Thus, the phonon frequencies $\omega_{q}$ are simply given by 


$$
\omega_{q}=\sqrt{\frac{\Theta_{q}}{M}} .
$$

\section{Nonanalytic terms}

For states close to the Fermi energy, the denominator in Eq. (7) goes to zero, resulting in the possible presence of singularities in the phonon dispersion, as we have shown in graphene and graphite. ${ }^{12}$ Similar singularities occur in the real part of Eq. (4) when the energy of the phonon matches an electronic transition (see, e.g., Eq. D.17 in Ref. 66). To investigate such singularities in SWNTs it is convenient to split the dynamical matrix into an analytic and a nonanalytic component,

$$
\Theta_{q}=\Theta_{q}^{\mathrm{an}}+\widetilde{\Theta}_{q} .
$$

The nonanalytic terms in the dynamical matrix of metallic tubes can be obtained by restricting the integral in the first term of Eqs. (4) and (7) to a set of $k$ points very close to the Fermi vector, and by limiting the sum over the electronic bands, taking into account only transitions between the bands crossing the Fermi point. Labeling with $L$ the electronic band with a positive slope in Fig. 2, and $R$ that with negative slope, from Eqs. (4) and (7) we obtain

$$
\begin{aligned}
\widetilde{\Theta}_{q}= & \frac{2 A_{\Gamma / K} T}{2 \pi} \sum_{m, n=L, R} \int_{-\bar{k}}^{\bar{k}}\left|D_{\left(K+k^{\prime}+q\right) n,\left(K+k^{\prime}\right) m}\right|^{2} \\
& \times \frac{f_{K+k^{\prime}, m}-f_{K+k^{\prime}+q, n}}{\epsilon_{K+k^{\prime}, m}-\epsilon_{K+k^{\prime}+q, n}+\hbar \omega_{q}+i \gamma} d k^{\prime},
\end{aligned}
$$

if dynamic effects are included. $\bar{k}$ has a small but finite value. In this case, divergencies occur when $\epsilon_{K+k^{\prime}, m}$ $-\epsilon_{K+k^{\prime}+q, n} \pm \hbar \omega_{q}=0$.

Within the static approximation we obtain

$$
\begin{aligned}
\widetilde{\Theta}_{q}= & \frac{2 A_{\Gamma / K} T}{2 \pi} \sum_{m, n=L, R} \int_{-\bar{k}}^{\bar{k}} \frac{f_{K+k^{\prime}, m}-f_{K+k^{\prime}+q, n}}{\epsilon_{K+k^{\prime}, m}-\epsilon_{K+k^{\prime}+q, n}} \\
& \times\left|D_{(K+k+q) n,\left(K+k^{\prime}\right) m}\right|^{2} d k^{\prime} .
\end{aligned}
$$

Here divergencies occur when $\epsilon_{K+k^{\prime}, m}=\epsilon_{K+k^{\prime}+q, n}$.

For both Eq. (10) and Eq. (11), divergences occur for $q$ $\sim 0$ or $q \sim K$ (Fig. 2). In the first case, $A_{\Gamma}=2$, in the second case $A_{K}=1$. Note that the distinction between the analytical and nonanalytical part of $\Theta_{q}$ is operative, not physical. In fact, $\widetilde{\Theta}_{q}$ contains all the divergent terms of $\Theta_{q}$ but not only them. The cut between $\widetilde{\Theta}_{q}$ and $\Theta_{q}^{\text {an }}$ depends on $\bar{k}$.

\section{Curvature and confinement}

The differences between graphene and a SWNT can be described in terms of curvature and confinement. Curvature effects are due to the distortion of the C-C bonds in the SWNT geometry and in a change of the bond character with respect to planar graphene. Confinement effects stem from the reduced dimensionality and are due to the quantization of the electronic wave functions along the SWNT circumference.
In this paper we concentrate on the description of the effects of confinement and curvature on the dynamical matrix and on the phonon frequencies of SWNTs. Considering the effects of curvature and confinement as perturbations on the dynamical matrix of graphene $\hat{\Theta}_{G}$, at first order,

$$
\hat{\Theta}_{T}=\hat{\Theta}_{G}+\hat{\Theta}_{\text {curv }}+\hat{\Theta}_{\text {conf }},
$$

where $\hat{\Theta}_{T}$ is the dynamical matrix of a SWNT and $\hat{\Theta}_{\text {curv }}$ and $\hat{\Theta}_{\text {conf }}$ account for curvature and confinement. The effects of pure confinement will be calculated in Secs. IV and V. The effects of curvature will be evaluated in Sec. VI.

\section{ELECTRON-PHONON COUPLING}

In Ref. 10 we have shown that the EPC of SWNT of arbitrary chirality is weakly affected by the tube curvature, and that it can be computed by folding the graphene EPC. Neglecting curvature, the EPC of a $(n, m)$ SWNT is related to that of graphene by the ratio of the unit cells,

$$
\left|D^{2}\right|\left|\mathbf{C}_{h} \times \mathbf{T}\right|=\left|\widetilde{D}^{2}\right| \frac{a_{0}^{2} \sqrt{3}}{2},
$$

where $D$ is the EPC of a $(n, m)$ SWNT; $\widetilde{D}$ is the graphene EPC; $\mathbf{C}_{h} \times \mathbf{T}$ the area of the unrolled tube unit cell; $a_{0}$ is the lattice parameter of graphene, and $a_{0}^{2} \sqrt{3} / 2$ is the graphene unit cell area. This formula is a simple consequence of electron and phonon normalization in the two different unit cells. Its derivation is presented in Appendix A.

Since $\mathbf{T}$ and $\mathbf{C}_{h}$ are perpendicular, $\left|\mathbf{C}_{h}\right|=\pi d$, being $d$ the tube diameter, from Eq. (13), the EPC of a SWNT is

$$
\left|D^{2}\right|=\frac{a_{0}^{2} \sqrt{3}}{2 T \pi d}\left|\widetilde{D}^{2}\right| .
$$

Using Eq. (14) in Eqs. (10) and (11), it is possible to obtain the expression for the nonanalytic part of the dynamical matrix within zone folding. Equation (10) becomes

$$
\begin{aligned}
\widetilde{\Theta}_{q}= & \frac{A_{\Gamma / \mathbf{K}} a_{0}^{2} \sqrt{3}}{2 \pi^{2} d} \sum_{m, n=L, R}\left|\widetilde{D}_{\left(\mathbf{K}+\mathbf{k}^{\prime}+\mathbf{q}\right) n,\left(\mathbf{K}+\mathbf{k}^{\prime}\right) m}\right|^{2} \\
& \times \int_{-\bar{k}}^{\bar{k}} \frac{f_{\mathbf{K}+\mathbf{k}^{\prime}, m}-f_{\mathbf{K}+\mathbf{k}^{\prime}+\mathbf{q}, n}}{\epsilon_{\mathbf{K}+\mathbf{k}^{\prime}, m}-\epsilon_{\mathbf{K}+\mathbf{k}^{\prime}+\mathbf{q}, n}+\hbar \omega_{\mathbf{q}}+i \gamma} d k^{\prime},
\end{aligned}
$$

while Eq. (11) becomes

$$
\begin{aligned}
\widetilde{\Theta}_{q}= & \frac{A_{\Gamma / \mathbf{K}} a_{0}^{2} \sqrt{3}}{2 \pi^{2} d} \sum_{m, n=L, R}\left|\tilde{D}_{\left(\mathbf{K}+\mathbf{k}^{\prime}+\mathbf{q}\right) n,\left(\mathbf{K}+\mathbf{k}^{\prime}\right) m}\right|^{2} \\
& \times \int_{-\bar{k}}^{\bar{k}} \frac{f_{\mathbf{K}+\mathbf{k}^{\prime}, m}-f_{\mathbf{K}+\mathbf{k}^{\prime}+\mathbf{q}, n}}{\epsilon_{\mathbf{K}+\mathbf{k}^{\prime}, m}-\epsilon_{\mathbf{K}+\mathbf{k}^{\prime}+\mathbf{q}, n}} d k^{\prime} .
\end{aligned}
$$

Note that, since in zone folding the electronic states of the tube are mapped onto those of graphene, all the wave vectors in Eqs. (15) and (16) are now vectorial quantities, and not scalar as in Eqs. (10) and (11). The integration is performed along the line of $\mathrm{ZF}_{(n, m)}$ passing through $\mathbf{K}$. Equations (15) and (16) also show that the dependence of $\widetilde{\Theta}_{\mathbf{q}}$ on the trans- 
TABLE I. EPC $|\widetilde{D}|^{2}$ in SWNTs for phonons corresponding to the graphene $\boldsymbol{\Gamma}-E_{2 g}$ (LO or TO) and $\mathbf{K}-A_{1}^{\prime}$ phonons. Electronic states are $L$ and $R$ bands near the Fermi energy (see Fig. 2). $\left\langle D_{\Gamma}^{2}\right\rangle_{F}$ $=45.60 \mathrm{eV} \AA^{-2}$ and $\left\langle D_{\mathbf{K}}^{2}\right\rangle_{F}=92.05 \mathrm{eV}^{-2}$, from Refs. 10 and 12 .

\begin{tabular}{cccc}
\hline \hline & LO & TO & K \\
\hline$\left|\widetilde{D}_{L R}\right|^{2}=\left|\widetilde{D}_{R L}\right|^{2}$ & $2\left\langle D_{\mathbf{\Gamma}}^{2}\right\rangle_{F}$ & 0 & $2\left\langle D_{\mathbf{K}}^{2}\right\rangle_{F}$ \\
$\left|\widetilde{D}_{L L}\right|^{2}=\left|\widetilde{D}_{R R}\right|^{2}$ & 0 & $2\left\langle D_{\Gamma}^{2}\right\rangle_{F}$ & $2\left\langle D_{\mathbf{K}}^{2}\right\rangle_{F}$ \\
\hline \hline
\end{tabular}

lational vector $\mathbf{T}$ disappears. This implies that, for metallic tubes, any dependence on tube chirality is lost as well, and all the information on tube geometry is given by the $\frac{1}{d}$ dependence only.

As already stated, the relevant EPCs correspond to the graphene phonons at $\boldsymbol{\Gamma}$ and $\mathbf{K}$, calculated between electronic states $L$ and $R$ near the Fermi energy (Fig. 2). In Ref. 12 it was shown that the only optical phonons of graphene with a non-negligible EPC are the $E_{2 g}$ phonon at $\boldsymbol{\Gamma}$ and the $A_{1}^{\prime}$ at $\mathbf{K}$. Thus, throughout the paper we will consider only the SWNT phonons corresponding to the graphene $\Gamma-E_{2 g}$ and to the $\mathbf{K}-A_{1}^{\prime}$. For simplicity, we will label them $\boldsymbol{\Gamma}$ (TO or LO) and $\mathbf{K}$. For these phonons, in Ref. 12 it is shown that the EPC can be considered independent from $k$ and assumes the values of Table I.

The EPC of graphene (and, thus, of SWNTs) are defined in literature in different ways: (i) the derivative of the hopping integral with respect to the C-C distance $(\eta),{ }^{8,41-43}$ (ii) the EPC matrix element $D$ of Eq. (5), (iii) $D$ times the phonon characteristic length, $(g) .{ }^{12}$ The values of $\eta, D$, and $g$ are related by the following expressions: ${ }^{10,12}$

$$
|D|^{2}=9 / 2 \eta^{2} \quad \text { and }|g|=|D| \sqrt{\frac{\hbar}{2 M \omega_{\mathbf{q}}}} .
$$

The relation between $\eta$ and $D$ is obtained by means of a first neighbors tight-binding model. ${ }^{67}$

A variety of contrasting EPC values are reported in literature. Table II compares literature results for the graphene EPC with those we experimentally measured and calculated

TABLE II. Comparison between calculated and experimental EPCs for the $\Gamma$ LO phonon, in its equivalent $\eta,|D|^{2}$, and $|g|^{2}$ expressions. All data in the literature were calculated with tight binding. The relation between $\eta$ and $D$ is obtained by means of a first neighbors tight-binding model.

\begin{tabular}{lccc}
\hline \hline & $\eta\left(\mathrm{eV} \AA^{-1}\right)$ & $\begin{array}{c}\left|\tilde{D}_{L R}\right|^{2} \\
\left(\mathrm{eV}^{2} \AA^{-2}\right)\end{array}$ & $\begin{array}{c}\left|\widetilde{g}_{L R}\right|^{2} \\
\left(\mathrm{eV}^{2}\right)\end{array}$ \\
\hline Mahan (Ref. 43) & $4.8-6.2$ & $103-172$ & $0.092-0.15$ \\
Park (Ref. 8) & 6 & 164 & 0.146 \\
Jiang (Ref. 41) & 1.94 & 17 & 0.015 \\
Perebeinos (Ref. 42) & 5.29 & 126 & 0.112 \\
Koswatta (Ref. 71) & 6.00 & 162 & 0.144 \\
DFT (Ref. 70) & 4.57 & 94 & 0.083 \\
Experimental (Ref. 12) & 4.50 & 91 & 0.081 \\
\hline \hline
\end{tabular}

in Refs. 12 and 70. This shows significant discrepancies between previous works and experimental EPCs, which, on the contrary, are in excellent agreement with our DFT calculations.

\section{CONFINEMENT EFFECTS WITHIN STATIC DFPT}

In this section we present a zone folding technique for the calculation of pure confinement effects on the phonon frequencies of SWNTs. All the results and calculations performed in this section are obtained within the static approximation.

\section{A. Electron and phonon zone folding}

A simple and effective method exists to calculate the SWNTs electronic structure taking into account confinement only. Indeed, in Sec. II A, it was shown that it is possible to fold the wave vectors of graphene into those of a $(n, m)$ SWNT. This technique can be used to obtain the electronic band structure of SWNTs from that of graphene., ${ }^{4,46,47} \mathrm{We}$ refer to this technique as electron zone folding (EZF). Using EZF it is possible to obtain the complete electronic structure of any SWNT doing the calculation just on the two atoms unit cell of graphene. EZF relies on the exact correspondence between the unrolled SWNT unit cell and graphene, therefore all the effects of curvature are completely neglected. Despite the simplicity of this technique, for SWNTs with $d$ $>0.8 \mathrm{~nm}$, the electronic structure calculated using EZF does not significantly differ from that calculated by DFT on the SWNT unit cell. ${ }^{40}$

The same procedure used to fold electrons can be used to fold phonons. ${ }^{4,5,18,38}$ We refer to this as phonon zone folding $(\mathrm{PZF})$. As already mentioned, KA affect graphene and metallic SWNTs differently, and do not affect semiconducting SWNTs at all. Thus, PZF is not suitable for the description of the phonon dispersion of SWNTs close to the anomalies.

\section{B. Phonon calculation within EZF}

Even if PZF cannot be used for the description of the Kohn anomalies, this result can still be achieved by means of a folding approach. Equation (7) shows that the dynamical matrix of a SWNT depends on its electronic structure through (i) the electronic charge density and (ii) an integral of the electronic states energies over the BZ. Numerically, both these quantities are obtained by performing discrete sums on a set of $k$ points over the tube BZ. These sums can be replaced by sums on points of $\mathrm{ZF}_{(n, m)}$ in the twodimensional graphene BZ. The evaluation of $\Theta_{q}$ within EZF completely neglects the effects of curvature. Thus, using Eq. (12), we find that SWNT dynamical matrices computed within EZF are in the form

$$
\hat{\Theta}_{T}^{\text {flat }}=\hat{\Theta}_{G}+\hat{\Theta}_{\text {conf }} .
$$

In this section, we use DFPT to calculate the SWNT dynamical matrices within EZF. We refer to these calculations as EZF-DFT. 
A further increase in the speed of calculations can be obtained from an appropriate choice of the BZ sampling. The graphene Fermi surface consists of the two inequivalent points $\mathbf{K}$ and $\mathbf{K}^{\prime}$. If the $\mathrm{ZF}_{(n, m)}$ lines cross these points, the tube is metallic, otherwise it is semiconducting. The $\pi$ and $\pi^{*}$ bands of graphene near the Fermi points have a conic shape, so the electronic bands of metallic SWNTs close to $\mathbf{K}$ and $\mathbf{K}^{\prime}$ are linear, and $\epsilon_{k^{\prime}}= \pm \beta k^{\prime}$. This implies that for metallic tubes the integral in Eq. (7) contains terms that diverge like $1 / k$. These singularities are responsible for the KA onset, and must be properly described. The most efficient way to numerically integrate $1 / k$ divergences in one dimension is to use a logarithmic sampling. ${ }^{72}$ Thus, to optimize the efficiency of our calculations, we sample the EZF lines with evenly spaced grids of $k$ points in regions away from the Fermi surface, and with denser logarithmic grids when crossing $\mathbf{K}$ or $\mathbf{K}^{\prime}$. Details on the $k$-point sampling used for EZFDFT calculations are reported in Table III.

\section{Comparison to other computational techniques}

It is important to discuss how our EZF-DFT results compare to other computational approaches in literature. These can be divided in the following categories: PZF ${ }^{38}$ tight binding, ${ }^{15,28-30,73}$ symmetry adapted methods, ${ }^{31,35,36,74}$ and DFT. ${ }^{17-20,27,32,51}$

The advantages of EZF-DFT over PZF have already been discussed in the preceding sections. The advantage of EZFDFT over conventional DFT is the possibility of using extremely dense $k$-point grids and to converge calculations for a smaller $T_{e}$. This is evident from a survey of previous DFT calculations. We start examining the DFT approach used in Refs. 17, 18, 27, and 51. These calculations were performed on the real tube unit cell. Thus the use of dense $k$-point grids to converge the phonon frequency at realistic $T_{e}$ was computationally extremely expensive. Calculations of Refs. 27 and 51 correspond to BZ sampling of at most three nonequivalent $k$ points in the unit cell. The comparison between the number of $k$ points used in Refs. 27 and 51 and those used in this and other works, seriously challenges the convergence of the calculations in Refs. 27 and 51.

References 17 and 18 used much denser $k$-point sampling (up to 57) and smeared electrons with an Hermite-Gauss function at a fictitious ${ }^{75}$ electronic temperature $T_{f}=1160 \mathrm{~K}$. In principle, these calculations should reproduce the $T_{e}=0$ results, showing PD in metallic tubes. However, these authors observed no PD, but just a softening of the phonon frequencies. This happens because their chosen value of $T_{f}$ is too large to reach the limit of $T_{e} \rightarrow 0$, and describes the system at a finite, but undefined, value of $T_{e}$. Calculations at lower $T_{e}$ were prevented by the huge CPU time requirements. DFT calculations at lower values of $T_{e}$ have been reported only for extremely small SWNTs in Refs. 19 and 20. Here, they calculated the phonon dispersion of SWNTs with $d \sim 4 \AA$ at $T_{e}$ as low as $137 \mathrm{~K}$, showing the presence of $\mathrm{KA}$ and PD. The comparison between the $k$-points sampling and the electronic temperature in literature and those used in this paper is reported in Table III.

As we will show in the next sections, the calculations of the phonon dispersion of large SWNTs at $T_{e}$ as low as $4 \mathrm{~K}$
TABLE III. $k$-points sampling used in our EZF-DFT calculations and data from other authors. The number of $k$ points $n_{k}$ refers to the number of nonequivalent points the tube BZ. These $n_{k}$ points fold into $n_{k} N_{c}$ points in the graphene BZ, $N_{c}$ being the number of unit cells of graphene present in the tube unit cell. The column $T_{e}$ is the electronic temperature used in the calculations (in Kelvin). Values marked with * correspond to calculations where electrons are smeared using a Hermite-Gauss instead of the Fermi-Dirac function. The $M / S$ column specifies the metallic or semiconducting nature of the tubes. The ln column specifies if a logarithmic grid is used.

\begin{tabular}{|c|c|c|c|c|c|}
\hline Tube & Reference & $T_{e}$ & $n_{k}$ & $M / S$ & $\ln$ \\
\hline$(n, n)$ & This work & 4 & 302 & $M$ & $y$ \\
\hline$(n, n)$ & This work & 77 & 91 & $M$ & $y$ \\
\hline$(n, n)$ & This work & 315 & 71 & $M$ & $y$ \\
\hline$(n, n)$ & This work & 3150 & 51 & $M$ & $y$ \\
\hline$(n, 0)$ & This work & 4 & 108 & $M$ & $y$ \\
\hline$(n, 0)$ & This work & 77 & 38 & $M$ & $y$ \\
\hline$(n, 0)$ & This work & 315 & 28 & $M$ & $y$ \\
\hline$(n, 0)$ & This work & 3150 & 18 & $M$ & $y$ \\
\hline$(n, 0)$ & This work & & 30 & $S$ & $n$ \\
\hline$(n, m)$ & This work & & $>\frac{2 \pi}{0.12 T}$ & $S$ & $n$ \\
\hline$(5,2)$ & This work & 315 & 26 & $M$ & $y$ \\
\hline$(12,3)$ & This work & 315 & 48 & $M$ & $y$ \\
\hline$(16,1)$ & This work & 315 & 23 & $M$ & $y$ \\
\hline$(20,14)$ & This work & 315 & 19 & $M$ & $y$ \\
\hline$(15,6)$ & This work & 315 & 18 & $M$ & $y$ \\
\hline$(14,8)$ & This work & 315 & 47 & $M$ & $y$ \\
\hline$(n, n)$ & Ref. 18 & $* 1160$ & 57 & $M$ & $n$ \\
\hline$(n, 0)$ & Ref. 18 & *1160 & 33 & $M$ & $n$ \\
\hline$(n, 0)$ & Ref. 18 & $* 1160$ & 13 & $S$ & $n$ \\
\hline$(n, n)$ & Ref. 27 & & 3 & $M$ & $n$ \\
\hline$(10,0)$ & Ref. 27 & & 2 & $S$ & $n$ \\
\hline$(8,4)$ & Ref. 27 and 51 & & 1 & $S$ & $n$ \\
\hline$(9,3)$ & Ref. 51 & & 1 & $S$ & $n$ \\
\hline$(3,3)$ & Ref. 19 & *1096 & 129 & $M$ & $n$ \\
\hline$(3,3)$ & Ref. 19 & ${ }^{*} 137$ & & $M$ & $n$ \\
\hline$(n, n)$ & Ref. 20 & 300 & & $M$ & $y$ \\
\hline$(5,0)$ & Ref. 20 & 300 & & $M$ & $y$ \\
\hline
\end{tabular}

can be easily done by means of EZF-DFT. This is achieved at the price of neglecting the effects of curvature, which prevents EZF-DFT to be used for the study of the radial breathing modes, and results in inaccuracies in the determination of the phonon frequencies of those tubes for which curvature effects are not negligible. This point will be discussed in more depth in Sec. VI.

EZF-DFT has also to be compared with tight binding and symmetry adapted calculations. Non-ab initio techniques, such as tight binding, offer another way to overcome the extremely large CPU time required by DFT. Most TB models are unable to describe KA and PD. ${ }^{28-30}$ A detailed study of EPC and $T_{e}$ effects on SWNT phonon within TB was reported by Barnett et al. ${ }^{15,73}$ This study investigated KA and 


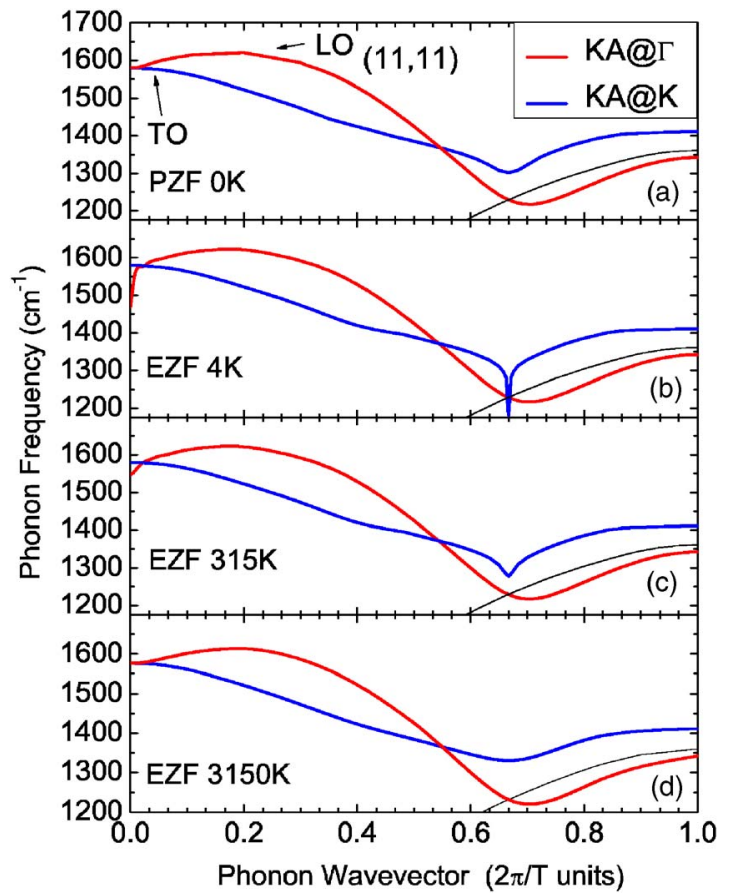

FIG. 3. (Color online) Phonons of the $(11,11)$ metallic SWNT calculated using (a) phonon zone folding (PZF), (b) electronic zone folding (EZF-DFT) at $T_{e}=4 \mathrm{~K}$, (c) $315 \mathrm{~K}$, and (d) $3150 \mathrm{~K}$. Branches affected by (red) KA at $\boldsymbol{\Gamma}$, (blue) KA at $\mathbf{K}$.

PD in SWNTs, but the results are in contrast with those obtained by DFT. ${ }^{20}$ Another possibility for the efficient calculations of phonons in SWNTs is the development of symmetry based force constants methods ${ }^{36}$ and lattice dynamic models based on the screw symmetry of SWNTs. ${ }^{31,35,74}$ So far, only TB implementations of symmetry adapted techniques were applied to the calculation of the vibrational properties of SWNTs, which thus may lack the precision of DFT. The implementation of screw symmetries into a DFT scheme would be highly desirable, but would require writing a new dedicated code. On the contrary, another advantage of EZF-DFT is that it can be performed with the already existing packages. Finally, it is very important to stress that all the techniques presented in this section rely on the static approximation, and thus neglect dynamic effects.

\section{Numerical results}

In this section, we use EZF-DFT to perform a systematic study of the phonon dispersion of SWNTs, paying particular attention to the description of KA and PD. Calculations are performed within the static approximation.

\section{Phonon dispersions}

Here we use EZF-DFT to calculate phonons of SWNTs of different diameters and chiralities, focusing on diameter and $T_{e}$ dependence of the KA induced phonon softening.

We start with the comparison of phonon zone folding and EZF-DFT for metallic tubes. Figure 3 compares the phonons of a metallic $(11,11)$ tube calculated using PZF with those obtained by EZF-DFT. To improve readability, we only plot

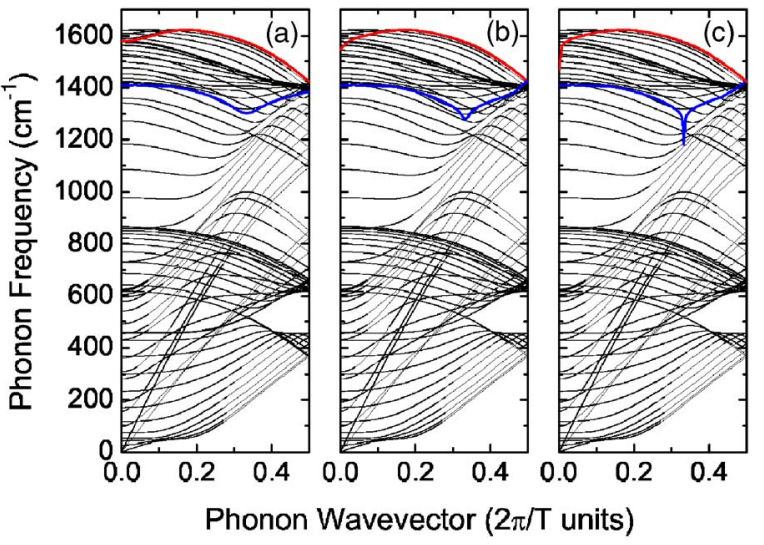

FIG. 4. (Color online) Comparison of the complete phonon dispersion of a $(11,11)$ tube calculated using (a) PZF, (b) EZF-DFT at $T_{e}=315 \mathrm{~K}$, (c) EZF-DFT at $T_{e}=4 \mathrm{~K}$. The three calculations differ only for the branches affected by KA.

phonon branches corresponding to the lines of $\mathrm{ZF}_{(11,11)}$ crossing $\boldsymbol{\Gamma}$ and $\mathbf{K}$. All PZF and EZF-DFT calculations are performed using DFPT. ${ }^{44}$ The parameters for PZF calculations are the same as those used for the calculation of graphite in Ref. 12.

PZF calculations use the phonons of graphene, thus (i) at $\Gamma$ no LO-TO splitting is observed, and (ii) the kinks and the phonon softening caused by KA at $\boldsymbol{\Gamma}$ and $\mathbf{K}$ are the same as those calculated in Ref. 12. Since the phonon frequencies of graphene converge at an electronic temperature $T_{e}$ $\sim 3000 \mathrm{~K}$, no temperature dependence of the phonon frequencies calculated by PZF can be observed for $0 \mathrm{~K}<T_{e}$ $<3000 \mathrm{~K}$. EZF-DFT calculations show a number of fundamental differences with respect to PZF. Figures 3(b)-3(d) show that the phonon softening induced by $\mathrm{KA}$ at $\boldsymbol{\Gamma}$ and $\mathbf{K}$ strongly depends on $T_{e}$, with the phonon frequencies decreasing for decreasing $T_{e}$. Most importantly, we observe that at $\boldsymbol{\Gamma}$ only the LO mode is affected by KA. This causes the splitting of the LO-TO modes. As already stressed, this splitting cannot be observed using PZF. Due to the temperature dependence of the LO mode, the LO-TO splitting increases for decreasing $T_{e}$. For $T_{e}=3150 \mathrm{~K}$, the KA effects are completely removed, and the LO-TO splitting is zero. Finally, in Fig. 4, we compare the whole phonon dispersion of the $(11,11)$ tube computed using (a) PZF, (b) EZF-DFT at $T_{e}=4 \mathrm{~K}$, and (c) $T_{e}=315 \mathrm{~K}$. Figure 4 shows that PZF and EZF-DFT differ only for the phonon branches affected by $\mathrm{KA}$, and are indistinguishable for all other phonon branches. Thus, in the phonon dispersion of metallic SWNTs only the modes affected by KA depend on $T_{e}$, and PZF can be safely used for the calculation of all other phonon modes.

It is also interesting to compare PZF and EZF-DFT for semiconducting tubes. Figure 5 compares PZF and EZF-DFT for a semiconducting $(19,0)$ tube. This has the same diameter $(1.5 \mathrm{~nm})$ as the metallic $(11,11)$ in Fig. 3. As for Fig. 3, in Fig. 5 we only plot selected phonon branches, in particular those corresponding to the line of $\mathrm{ZF}_{(19,0)}$ crossing $\boldsymbol{\Gamma}$ and those corresponding to the two lines that lay most closely to K. KA cannot be present in semiconductors, thus no KA should be observed in the $(19,0)$ tube. Since $\mathrm{ZF}_{(19,0)}$ does 


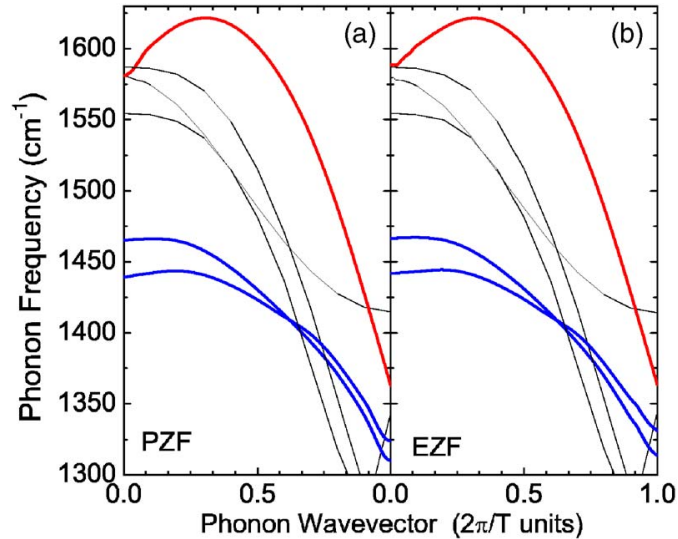

FIG. 5. (Color online) Phonons of the $(19,0)$ tube calculated using (a) PZF on a $q$-points grid sampling $\mathrm{ZF}_{(19,0)}$, (b) EZF-DFT. Branches that in graphene and metallic tubes would be affected by the KA anomaly at $\boldsymbol{\Gamma}$ are in red, while those closest to the KA at $\mathbf{K}$ in graphene are in blue.

not include $\mathbf{K}$, and the empty and occupied electronic states are separated by a gap $E_{g} \sim 0.5 \mathrm{eV}$, no temperature effects can be observed for $T_{e}<E_{g} / k_{B} \sim 6000 \mathrm{~K}$.

Figure 5 shows that the discrepancies between PZF and EZF-DFT are extremely reduced with respect to those of the metallic $(11,11)$ tube, the only noticeable difference being the absence of LO-TO splitting in the PZF calculations.

\section{LO-TO splitting}

Within EZF-DFT, the doubly degenerate $\boldsymbol{\Gamma}-E_{2 g}$ phonon of graphene splits for both metallic and semiconducting tubes. This splitting is a direct consequence of confinement in SWNTs.

Defining $\omega_{\mathrm{LO}(\mathrm{TO})}^{S(M)}$ as the phonon frequency of the LO (TO) mode at $\mathbf{q}=0$ for a semiconducting (metallic) SWNT, we also observe that $\omega_{\mathrm{TO}}^{M}>\omega_{\mathrm{LO}}^{M}$ while $\omega_{\mathrm{LO}}^{S}>\omega_{\mathrm{TO}}^{S}$. This is explained as follows. From Table I, the EPCs between $L$ and $R$ bands at $\boldsymbol{\Gamma}$ have a finite value for $\mathrm{LO}$ modes, and are null for TO modes. Since the EPC associated with TO phonons is null, from Eq. (11) we obtain that $\omega_{\mathrm{TO}}^{\mathrm{graph}}=\omega_{\mathrm{TO}}^{S}=\omega_{\mathrm{TO}}^{M}$, where $\omega_{\mathrm{TO}}^{\text {graph }}$ is the phonon frequency of the TO mode at $\boldsymbol{\Gamma}$ for graphene. On the other hand, LO modes have a finite EPC. Here it is important to remind that what determines the physical properties related to EPC is not the EPC per se, but the product of the EPC times the electronic density of states (DOS) (see also Appendix A). Close to the Fermi energy, for semiconducting SWNTs, the DOS is null, while for metallic SWNTs it is a constant, and for graphene is zero at Fermi energy $\left(\epsilon_{F}\right)$ and increases linearly for $\epsilon>\epsilon_{F}$ and $\epsilon<\epsilon_{F}$. It follows that $\operatorname{DOS}_{\epsilon_{F}}^{S}<\operatorname{DOS}_{\epsilon_{F}}^{\text {graph }}<D O S_{\epsilon_{F}}^{M}$, where the superscripts $S, M$, and graph refer, respectively, to semiconducting SWNTs, metallic SWNTs, and graphene, and the subscript $\epsilon_{F}$ indicates that the DOS is evaluated for energies $\epsilon$ close to the Fermi energy $\epsilon_{F}$. From Eq. (11), and using the same notation as the TO modes, we then obtain $\omega_{\mathrm{fO}}^{M}<\omega_{\mathrm{LO}}^{\text {graph }}<\omega_{\mathrm{LO}}^{S}$. Since in graphene we have $\omega_{\mathrm{LO}}^{\text {graph }}=\omega_{\mathrm{TO}}^{\text {graph }}$, it follows that $\omega_{\mathrm{LO}}^{S}$ $>\omega_{\mathrm{TO}}^{S}$ and $\omega_{\mathrm{LO}}^{M}<\omega_{\mathrm{TO}}^{S}$.

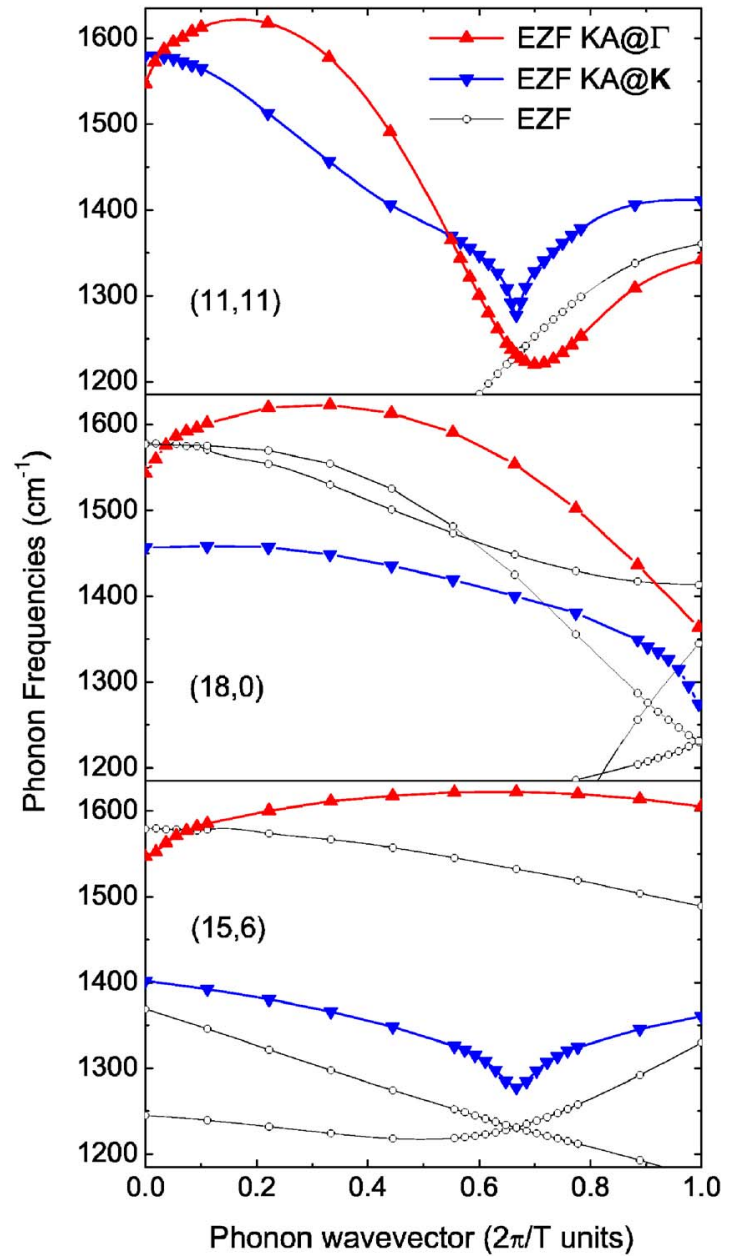

FIG. 6. (Color online) Phonons of the $(11,11),(18,0)$, and $(15,6)$ tubes calculated using EZF-DFT. All tubes have $d$ $\sim 1.5 \mathrm{~nm}$. Calculations are done at $T_{e}=315 \mathrm{~K}$. Phonon branches affected by KA at $\boldsymbol{\Gamma}$ are in red, those with KA at $\mathbf{K}$ are in blue.

With EZF-DFT, we also find that the atomic displacements of the modes deriving from the graphene $\boldsymbol{\Gamma}-E_{2 g}$ are parallel or perpendicular to $\mathbf{q}_{\|}$within an angle less than $3.25^{\circ}$. The effect of confinement is then, for any chirality, to split the $\boldsymbol{\Gamma}-E_{2 g}$ mode of graphene into almost exactly LO and TO modes.

\section{Diameter, electronic temperature, chirality and family dependence of phonon softening}

We now consider the dependence of the phonon dispersions on SWNT chirality, diameter and electronic temperature.

Figure 6 plots the phonon dispersions at $T_{e}=315 \mathrm{~K}$ for the $(11,11)$ armchair tube, the $(18,0)$ zigzag tube, and the $(15,6)$ chiral tube. All these tubes are metallic, and have a similar diameter of $1.5 \mathrm{~nm}$. Note that in SWNTs, defining $d_{R}=\operatorname{gcd}(2 n+m, 2 m+n), \mathbf{k}_{F}=0$ if $(n-m) / 3 d_{R}$ is not an integer [e.g., the $(18,0)$ tube], and $\mathbf{k}_{F}=1 / 3$, in $2 \pi / T$ units, if $(n-m) / 3 d_{R}$ is an integer [e.g., $(11,11)$ and $(15,6)$ tubes]. ${ }^{5}$ This explains the different KA positions in Fig. 6. We observe that, even if the shapes of the phonon dispersions are 


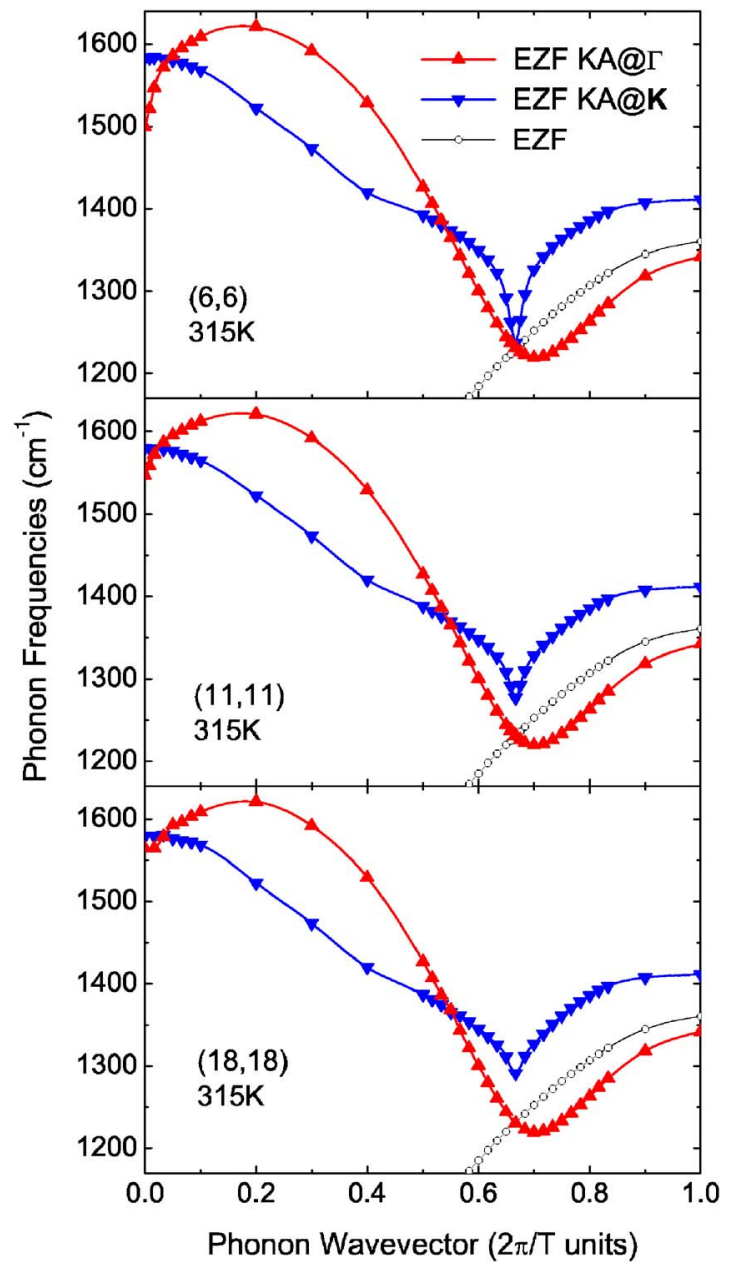

FIG. 7. (Color online) Phonons of the $(6,6),(11,11)$, and $(18,18)$ SWNTs calculated using EZF-DFT. Tube diameters are $0.8 \mathrm{~nm}, 1.5 \mathrm{~nm}$, and $2.4 \mathrm{~nm}$, respectively. $T_{e}$ is $315 \mathrm{~K}$. Phonon branches affected by KA at $\boldsymbol{\Gamma}$ are in red, while those with KA at $\mathbf{K}$ are in blue.

different, the frequency of the phonons corresponding to the graphene $\Gamma-E_{2 g \text {-LO }}$ and $\mathbf{K}-A_{1}^{\prime}$ are the same in all cases, and are significantly softened with respect to graphene. Thus, the phonon softening due to KA does not depend on chirality.

On the other hand, Figs. 7 and 4 clearly show that KAinduced phonon-softening strongly depends on diameter and $T_{e}$. Figure 7 compares the phonons for three different armchair tubes at the same $T_{e}$. It indicates that the smaller the diameter, the stronger the softening. Figure 4 compares phonons for the same $(11,11)$ tube at three different $T_{e}$. It shows that phonon softening increases for decreasing $T_{e}$. Note that, as for Figs. 3 and 5, Figs. 6 and 7 also plot only branches corresponding to $\mathrm{ZF}_{(n, m)}$ crossing $\boldsymbol{\Gamma}$ and $\mathbf{K}$.

Finally, due to the phonon softening induced by the KA, we observe that in metallic tubes of any chirality the LO branch always crosses the TO, in contrast with Ref. 76, where an anticrossing of the branches is predicted for chiral tubes.

Figure 8 plots the LO-TO phonon frequencies for a variety of chiral and achiral tubes, both metallic and semiconducting, in the diameter range $0.8-2.7 \mathrm{~nm}$ at $T_{e}=315 \mathrm{~K}$. As

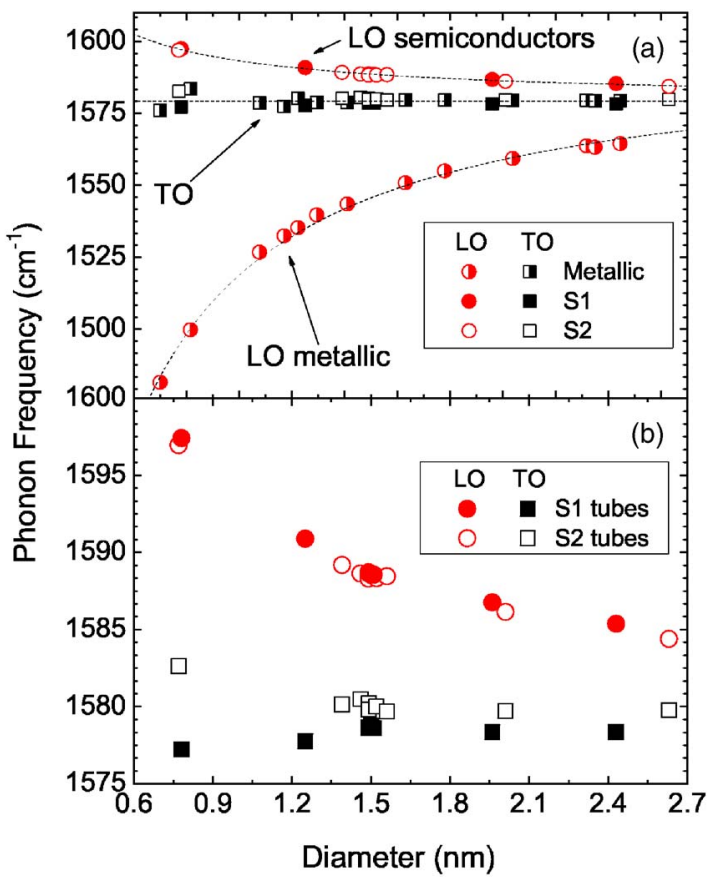

FIG. 8. (Color online) (a) Frequency of the LO and TO modes calculated by EZF-DFT at $T_{e}=315 \mathrm{~K}$. The LO phonons are in red; the TO phonons in black. Different symbols identify metallic and semiconducting $(S 1$ and $S 2)$ tubes. Dashed lines represent the fit of the EZF-DFT data, and correspond to the plot of Eq. (28). (b) LO and TO phonons of semiconducting tubes only. For $S 2$ tubes TO phonon is $\sim 2 \mathrm{~cm}^{-1}$ higher than in $S 1$ tubes. No family dependence is observed for the LO phonon.

expected, we observe no difference in the phonon frequencies for TO modes of metallic and semiconducting tubes. Furthermore, confinement does not induce any diameter dependence of the frequencies of the TO modes.

Due to the KA presence, LO frequencies are very different in metallic and semiconducting tubes. In metallic tubes there is a strong diameter dependence, with higher softening for decreasing diameter. This softening is entirely due to confinement. Chirality does not affect the LO phonons. This is shown in Fig. 9, which plots LO and TO modes for all semiconducting tubes with $d \simeq 1.5 \mathrm{~nm}$ as a function of chirality.

However, even if there is no dependence on the chiral angle for the LO modes, a family dependence is observed for the TO mode in semiconducting tubes. Indeed, Figs. 8(a) and 8(b) and Fig. 9 show that TO phonons are more scattered than LO. By labeling $S 1$ SWNTs with $\bmod (n-m, 3)=1$, and $S 2$ SWNTs with $\bmod (n-m, 3)=2$, it is possible to observe that the TO frequency in $S 1$ tubes is always $\sim 2 \mathrm{~cm}^{-1}$ smaller than in $S 2$ tubes. The TO frequency for metallic tubes is in between that of $S 1$ and $S 2$ tubes.

Figure 10 plots the LO phonon of metallic tubes as a function of $T_{e}$. Since no chirality dependence is observed for $T_{e}=315 \mathrm{~K}$, only armchair and zigzag tubes are considered. The LO softening increases for decreasing $T_{e}$, and the trend is stronger for smaller diameters.

We stress again, as in Sec. II D, that the temperature effects calculated here with EZF-DFT are related only to the electronic temperature. Therefore, for a direct comparison 


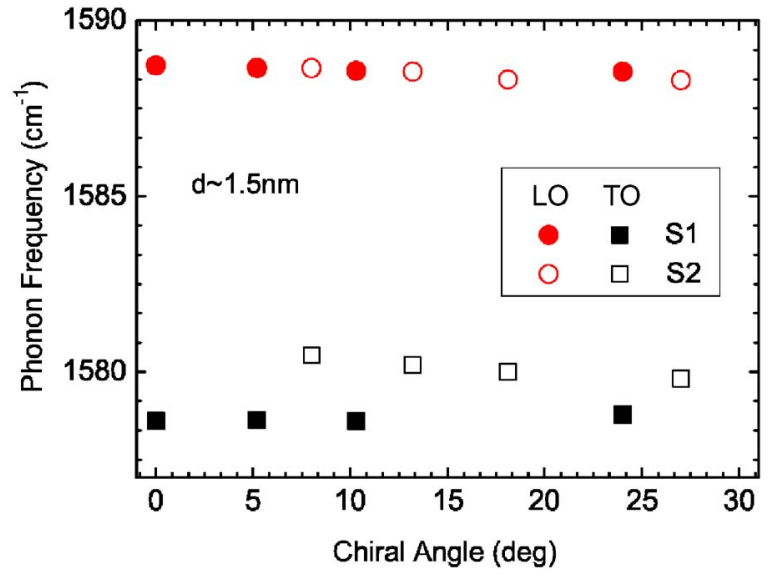

FIG. 9. (Color online) Calculated LO and TO modes obtained by EZF-DFT for the semiconducting tubes $(19,0),(18,2),(17,3)$, $(17,4),(16,5),(15,7),(13,9)$, and $(12,10)$, listed by increasing chiral angle. All tubes have diameter $d \sim 1.5 \mathrm{~nm}$. Filled symbols refer to $S 1$ tubes $[\bmod (n-m, 3)=1]$; open symbols to $S 2$ tubes $[\bmod (n-m, 3)=2]$. In $S 2$ tubes the TO mode frequency is $\sim 2 \mathrm{~cm}^{-1}$ higher than in $S 1$.

with experiments, the data in Fig. 10 need to be corrected with the anharmonic effects.

\section{E. Analytic results}

So far we relied on numerical DFPT calculations. We now show that the key results of the preceding section can be derived and explained by using an analytic model. From Eqs. (8) and (9), we have

$$
\omega_{q}^{2}=\frac{\Theta_{q}^{\mathrm{an}}}{M}+\frac{\widetilde{\Theta}_{q}}{M} .
$$

KAs are due to the presence of nonanalytic terms in the dynamical matrix and their shape is determined by $\widetilde{\Theta}_{\mathbf{q}}$, whose expression, within a static approach, is given by Eq. (16). $\widetilde{\Theta}_{\mathbf{q}}$ can be determined analytically for any $\mathbf{q}$ at $T_{e}$ $=0 \mathrm{~K}$, or at $\mathbf{q}=\boldsymbol{\Gamma}, \mathbf{K}$ for any $T_{e}$. This provides simple formu-

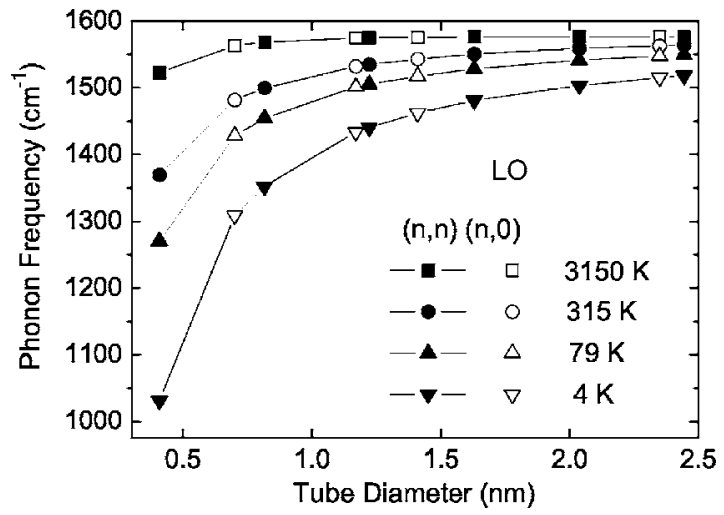

FIG. 10. Diameter dependence of LO mode in armchair and metallic zigzag SWNTs calculated by EZF-DFT for $T_{e}=4,79,315$, $3150 \mathrm{~K}$. las for the determination of the metallic-semiconducting transition associated with the PD and for the KA description in SWNTs.

\section{Kohn anomalies at $\mathrm{T}=0 \mathrm{~K}$ and Peierls distortions}

To determine the KA shape at zero temperature we set $T_{e}=0$ in Eq. (16). This gives $f_{\mathbf{k}}=1$ and $f_{\mathbf{k}}=0$ for states, respectively, below and above the Fermi energy. Equation (16) can then be integrated analytically to give

$$
\begin{gathered}
\widetilde{\Theta}_{\mathrm{LO} / \mathbf{K}}=\frac{A_{\Gamma / \mathbf{K}} \sqrt{3} a_{0}^{2} 2\left\langle D_{\Gamma / \mathbf{K}}^{2}\right\rangle_{F}}{\pi^{2} d \beta} \ln |q|+C_{\mathrm{LO} / \mathbf{K}}^{\prime}, \\
\widetilde{\Theta}_{\mathrm{TO}}=-\frac{A_{\Gamma} \sqrt{3} a_{0}^{2} 2\left\langle D_{\Gamma}^{2}\right\rangle_{F}}{\pi^{2} d \beta},
\end{gathered}
$$

where $\left\langle D_{\Gamma / \mathbf{K}}^{2}\right\rangle_{F}$ are defined in Table I and we assume the $L$ and $R$ bands to be linear with slope $\beta=5.25 \mathrm{eV} \AA$. More details on the integration are given in Appendix B. From Eq. (20), we observe that $\widetilde{\Theta}_{\text {TO }}$ does not depend on $q$. This explains the absence of KA for the TO mode. On the other hand, $\widetilde{\Theta}_{\mathrm{LO} / \mathbf{K}}$ has a logarithmic dependence on $q$, which explains the presence of KA in the LO branch. Using Eq. (19), we obtain

$$
\begin{aligned}
& \omega_{\mathrm{LO} / \mathbf{K}}^{2}=\frac{\alpha_{\Gamma / \mathbf{K}}}{d} \ln |q|+C_{\mathrm{LO} / \mathbf{K}}, \\
& \alpha_{\Gamma / \mathbf{K}}=\frac{2 \sqrt{3} A_{\Gamma / \mathbf{K}} a_{0}^{2}\left\langle D_{\Gamma / \mathbf{K}}^{2}\right\rangle_{F}}{\pi^{2} \beta M},
\end{aligned}
$$

where $q$ is measured from $\boldsymbol{\Gamma}$ for the KA at $\boldsymbol{\Gamma}$ and from $\mathbf{K}$ for the KA at $\mathbf{K} ; \quad \alpha_{\Gamma}=7.89 \times 10^{5} \mathrm{~cm}^{-2} \AA^{-1} ; \quad \alpha_{\mathbf{K}}=7.96$ $\times 10^{5} \mathrm{~cm}^{-2} \AA^{-1} ; C_{\mathrm{LO} / \mathrm{K}}$ account for all the nondivergent terms of the dynamical matrix.

According to Eq. (21), KA in SWNTs have a logarithmic shape. For the $\mathrm{LO}$ and $\mathbf{K}$ modes at $T_{e}=0 \mathrm{~K}, \omega_{\mathrm{q}}^{2}$ is negative, which gives imaginary phonon frequencies. This means that the lattice undergoes a permanent distortion, i.e., a Peierls distortion.

Figure 11 plots Eq. (21) for the KA at $T_{e}=0 \mathrm{~K}$ and compares it with EZF-DFT calculations in the limit $T_{e} \rightarrow 0 \mathrm{~K}$. Using Eq. (21), and fitting $C_{\mathrm{LO} / \mathrm{K}}$ to the EZF-DFT data, we obtain

$$
\begin{gathered}
\omega_{\mathrm{LO}}^{2}=5.3 \times 10^{4} \ln q+2.70 \times 10^{6} \mathrm{~cm}^{-2}, \\
\omega_{\mathrm{K}}^{2}=5.3 \times 10^{4} \ln q+1.94 \times 10^{6} \mathrm{~cm}^{-2} .
\end{gathered}
$$

From Fig. 11, Eq. (21) perfectly reproduces the phonon dispersions obtained by EZF-DFT.

\section{Electronic temperature dependence of Kohn anomalies and Peierls distortion temperature}

We now fix $\mathbf{q}=\boldsymbol{\Gamma}$ or $\mathbf{q}=\mathbf{K}$ and study the dependence of the phonon frequencies on $T_{e}$, which changes the occupation functions $f_{\mathbf{k}, n}$. For $\mathbf{q}=\boldsymbol{\Gamma}, \mathbf{K}$, assuming linear $L$ and $R$ bands with slope $\beta= \pm 5.52 \mathrm{eV} \AA$, remembering that $\mathbf{K}+\mathbf{K}=\mathbf{K}^{\prime}$, and setting $\epsilon_{F}=0$, we have 


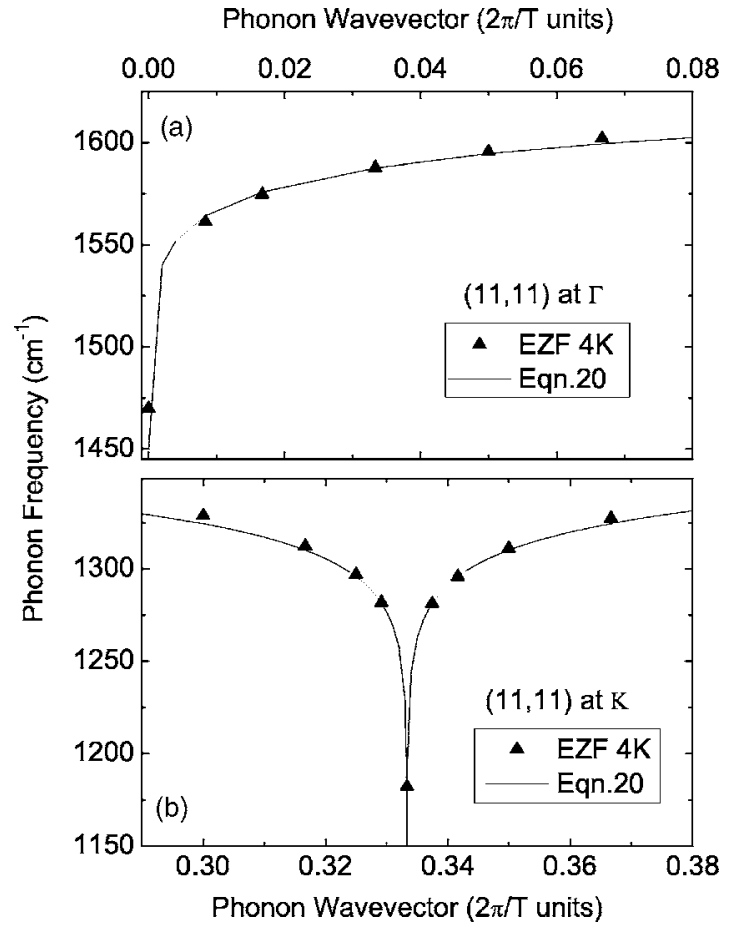

FIG. 11. KA at $\mathbf{\Gamma}$ and $\mathbf{K}$ for a $(11,11)$ tube. Triangles correspond to EZF-DFT at $T_{e}=4 \mathrm{~K}$. The solid line is the plot of Eq. (21).

$$
\begin{gathered}
\epsilon_{\left(\mathbf{K}+\mathbf{k}^{\prime}\right), L}=\epsilon_{\left(\mathbf{K}+\mathbf{k}^{\prime}+\Gamma\right), L}=\epsilon_{\left(\mathbf{K}+\mathbf{k}^{\prime}+\mathbf{K}\right), L}=k^{\prime} \beta, \\
\epsilon_{\left(\mathbf{K}+\mathbf{k}^{\prime}\right), R}=\epsilon_{\left(\mathbf{K}+\mathbf{k}^{\prime}+\Gamma\right), R}=\epsilon_{\left(\mathbf{K}+\mathbf{k}^{\prime}+\mathbf{K}\right), R}=-k^{\prime} \beta .
\end{gathered}
$$

The occupation factors $f_{\mathbf{k}, n}$ depend on $\mathbf{k}$ and $n$ only through $\epsilon_{n}(\mathbf{k})-\epsilon_{F}$. Defining $x=\beta k^{\prime} / T k_{B}$, and $\theta(x)=1 /\left(1+e^{x}\right)$, Eq. (16) becomes

$$
\widetilde{\Theta}_{\mathbf{\Gamma} / \mathbf{K}}=\frac{A_{\Gamma / \mathbf{K}} 2 \sqrt{3} a_{0}^{2}\left\langle D_{\Gamma / \mathbf{K}}^{2}\right\rangle_{F}}{\pi^{2} d \beta} \int_{0}^{\beta \bar{k} / T k_{B}} \frac{\theta(x)-\theta(-x)}{x} d x,
$$

where the bands' symmetry allows to replace $\int_{-\beta \bar{k} / T k_{B}}^{\overline{\beta k} / T k_{B}}$ with $2 \int_{0}^{\beta \bar{k} / T k_{B}}$. This gives

$$
\omega_{\mathrm{LO}, \mathbf{K}}^{2}(T)=\frac{\alpha_{\Gamma, \mathbf{K}}}{d} \ln \frac{T}{T_{0}}+C C_{\Gamma, \mathbf{K}},
$$

where $\alpha_{\mathbf{q}}$ is the same as in Eq. (21), $T_{0}$ is the electronic temperature for which the contributions of $\widetilde{\Theta}_{\mathbf{q}}$ are null, $C C_{\Gamma, \mathbf{K}}$ is the value of $\omega_{\Gamma / \mathbf{K}}^{2}$ at $T_{e}=T_{0}$, and contains all the contributions from $\Theta_{\mathbf{q}}^{\text {an }}$. Details on how Eq. (26) is derived from Eq. (16) are given in Appendix C. Equation (26) can be used to fit simultaneously all the phonon frequencies calculated by EZF-DFT as a function of $T_{e}$. The fit parameters are listed in Table IV.

The electronic temperature, $T_{\mathrm{PD}}$, for which a SWNT undergoes a Peierls distortion, can be obtained by setting $\omega_{\mathbf{q}}=0$ in Eq. (26), which leads to

$$
T_{\mathrm{PD}}=T_{0} e^{-d \cdot C C_{\boldsymbol{\Gamma}, \mathbf{K}} / \alpha_{\boldsymbol{\Gamma}, \mathbf{K}}}
$$

TABLE IV. Values of $T_{0}$ and $C C_{\Gamma, \mathbf{K}}$ obtained fitting the EZFDFT data for tubes calculated at $T_{e}=4 \mathrm{~K}, T_{e}=77 \mathrm{~K} T_{e}=315 \mathrm{~K} T_{e}$ $=3150 \mathrm{~K}$ and for $d$ in the range $0.8-2.4 \mathrm{~nm}$ with Eq. (26).

\begin{tabular}{lccc}
\hline \hline $\mathbf{q}$ & $T_{0}(\mathrm{~K})$ & $C C\left(\mathrm{eV}^{2}\right)$ & $C C\left(\mathrm{~cm}^{-2}\right)$ \\
\hline $\boldsymbol{\Gamma}$ & 9612 & 0.039 & $2.54 \times 10^{6}$ \\
$\mathbf{K}$ & 2646 & 0.027 & $1.76 \times 10^{6}$ \\
\hline \hline
\end{tabular}

As an example, Eq. (27) applied to the $(6,6)$ tube $(d$ $=0.8 \mathrm{~nm}$ ) gives $T_{\mathrm{PD}}=6.08 \times 10^{-8} \mathrm{~K}$ for the phonon at $\boldsymbol{\Gamma}$, and $T_{\mathrm{PD}}=0.5 \times 10^{-5} \mathrm{~K}$ for the phonon at $\mathbf{K} \cdot T_{\mathrm{PD}}$ exponentially decreases with the tube diameter. This implies that, unlike the ultrasmall tubes studied in Refs. 19 and 20, for the SWNTs generally used in experiments, $T_{\mathrm{PD}}$ is always smaller than $10^{-8} \mathrm{~K}$.

Equation (26) defines the diameter dependence of the LO phonon frequency of metallic tubes $\left(\omega_{\mathrm{LO}}^{M}\right)$ at any $T_{e}$, and can be used to fit the EZF-DFT data of Fig. 8. We observe that all the EZF-DFT data in Fig. 8 can be represented by very simple functions of the tube diameter. Indeed, neglecting the very small difference observed for the $S 1$ and the $S 2$ tubes, the frequency of the TO mode of both metallic and semiconducting tubes $\left(\omega_{\mathrm{TO}}\right)$ is diameter independent, while the calculated frequencies of the LO mode for semiconducting tubes $\left(\omega_{\mathrm{LO}}^{S}\right)$ are inversely proportional to the tube diameter. This can be summarized by the following equations:

$$
\omega_{\mathrm{LO}}^{M}=\eta_{M}+\frac{\nu_{M}}{d}, \quad \omega_{\mathrm{LO}}^{S}=\eta_{S}+\frac{\nu_{S}}{d}, \quad \omega_{\mathrm{TO}}=\xi,
$$

where $\quad \nu_{M}=-77.33 \mathrm{~cm}^{-1} \mathrm{~nm}, \quad \eta_{M}=1597 \mathrm{~cm}^{-1}, \quad \nu_{S}$ $=13.78 \mathrm{~cm}^{-1} \mathrm{~nm}$, and $\eta_{S}=\xi=1579 \mathrm{~cm}^{-1}$, which perfectly reproduces the phonon frequency we calculate for the $\boldsymbol{\Gamma}-E_{2 g}$ mode of graphene. The functional form of $\omega_{\mathrm{LO}}^{M}$ has been obtained from Eq. (26) using the relation $\sqrt{1+x} \sim 1+x / 2$. The expression for $\omega_{\mathrm{LO}}^{M}$ is thus completely analytical, but is valid only for $T_{e}=315 \mathrm{~K}$. On the other hand, the expressions for $\omega_{\mathrm{TO}}$ and $\omega_{\mathrm{LO}}^{S}$ are empirical, but are valid for any $T_{e}$. Equation (28) are plotted in Fig. 8.

\section{CONFINEMENT EFFECTS WITHIN DYNAMIC DFPT}

In Sec. II E, we argued the possible presence of dynamic effects for one-dimensional metallic systems. To the best of our knowledge, the dynamic nature of phonons has always been neglected in the SWNT literature, until we recently pointed this out. ${ }^{62}$ In this section, we show that dynamic effects are present in metallic SWNTs, and that they induce significant changes to the KA occurrence and shape. Therefore, they must be included to explain the experimental data. In fact, SWNTs are one of the first real materials for which is a significant difference between static and dynamic DFPT is detected.

If the static approximation is relaxed, the dynamical matrix is described by Eq. (4), and its nonanalytic part by Eq. (10). Assuming $T=0$, Eq. (10) can be solved by using a procedure similar to that used to obtain Eq. (20). For the LO phonon, assuming $\bar{k} \gg\left|q \pm \frac{\hbar \omega_{\mathrm{q}}}{\beta}\right|$, we obtain 


$$
\widetilde{\Theta}_{\mathrm{LO}}=\frac{A_{\Gamma} \sqrt{3} a_{0}^{2}\left\langle D_{\Gamma}^{2}\right\rangle_{F}}{\pi^{2} \beta d} \ln \frac{\left|\beta q+\hbar \omega_{\mathrm{LO}}\right|\left|\beta q-\hbar \omega_{\mathrm{LO}}\right|}{|2 \beta \bar{k}|^{2}},
$$

while for the TO,

$$
\widetilde{\Theta}_{\mathrm{TO}}=-\frac{A_{\Gamma} \beta q^{2} \sqrt{3} a_{0}^{2} 2\left\langle D_{\Gamma}^{2}\right\rangle_{F}}{\pi^{2} d\left[\beta^{2} q^{2}-\left(\hbar \omega_{\mathrm{TO}}\right)^{2}\right]},
$$

where $\omega_{\text {LO-TO }}$ is the phonon frequency of a phonon of wave vector $q \sim \Gamma$ belonging to the same branch of the LO-TO phonon at $q=0$. These equations mark a major departure from the previous results. $\tilde{\Theta_{q}}$ in the dynamic approach is very different from the static case. Note that the static case [Eq. (20)] is immediately recovered setting $\hbar \omega_{\mathrm{LO}-\mathrm{TO}}=0$ in Eqs. (29) and (30).

The dynamic effects are qualitatively very different for LO and TO phonons. Equation (29) diverges for $q=\hbar \omega_{\mathbf{q}} / \beta$, and not for $q=0$ as in the static case. This gives the first major result that the KA for the LO mode is not at $\Gamma$, contrary to what is predicted by any static approach, but at $q$ $= \pm \hbar \omega_{\mathrm{LO}} / \beta$.

The dynamic effects are even bigger for the TO phonons. Equation (30) shows that $\widetilde{\Theta}_{\mathrm{TO}}$ is zero for $q=0$, but diverges to $+\infty$ for $q \rightarrow\left(\hbar \omega_{\mathrm{TO}} / \beta\right)^{-}$and to $-\infty$ for $q \rightarrow\left(\hbar \omega_{\mathrm{TO}} / \beta\right)^{+}$. This is quite different from the static case, where $\widetilde{\Theta}_{\mathrm{TO}}$ is a negative constant. This results in a significant TO upshift with respect to the static case. This can be described by a simple equation, the derivation of which is given in Appendix D,

$$
\omega_{\mathrm{TO}}^{\mathrm{dyn}}-\omega_{\mathrm{TO}}^{\mathrm{stat}} \sim \frac{25}{d},
$$

where the frequency difference is in $\mathrm{cm}^{-1}$ and $d$ in $\mathrm{nm}$.

To summarize, the static approach: (i) fails to describe the position of the KA for the LO mode, (ii) underestimates the TO phonon frequency, (iii) misses the TO Kohn anomaly at $q=\hbar \omega_{\mathrm{TO}} / \beta$.

The phonon dispersion of metallic SWNTs close to the KAs is obtained by adding the contributions from the analytic and the nonanalytic part of the dynamical matrix. The contribution of the nonanalytic part is given by the numerical integration of Eq. (10). Due to the presence of the phonon energy in the expression of $\widetilde{\Theta}_{q}$, Eq. (10) must be solved self-consistently. The contribution from $\Theta_{q}^{\mathrm{an}}$ is easily obtained from EZF-DFT. The definitions of $\widetilde{\Theta}_{q}$ and $\Theta_{q}^{\text {an }}$ depend on the value chosen for $\bar{k}$. In our calculations, we chose $\bar{k}$ such that $\bar{k} \beta=1.0 \mathrm{eV}$.

Figure 12 compares the phonon frequencies of the $\mathrm{LO}$ and TO branches of an $(11,11)$ tube calculated using EZF-DFT with those obtained from the numerical integration of Eqs. (16) and (10). Figure 12 shows major differences between the dynamic and the static approach in the region of the phonon dispersions affected by KA. As expected, in the dynamic case, the KA in the LO branch is shifted from $q=0$ to a finite wave vector. This causes a significant change close to $\boldsymbol{\Gamma}$. However, Fig. 12 also indicates that these changes are confined to a very small BZ region. Indeed, for $\mathbf{q}>0.02$ in

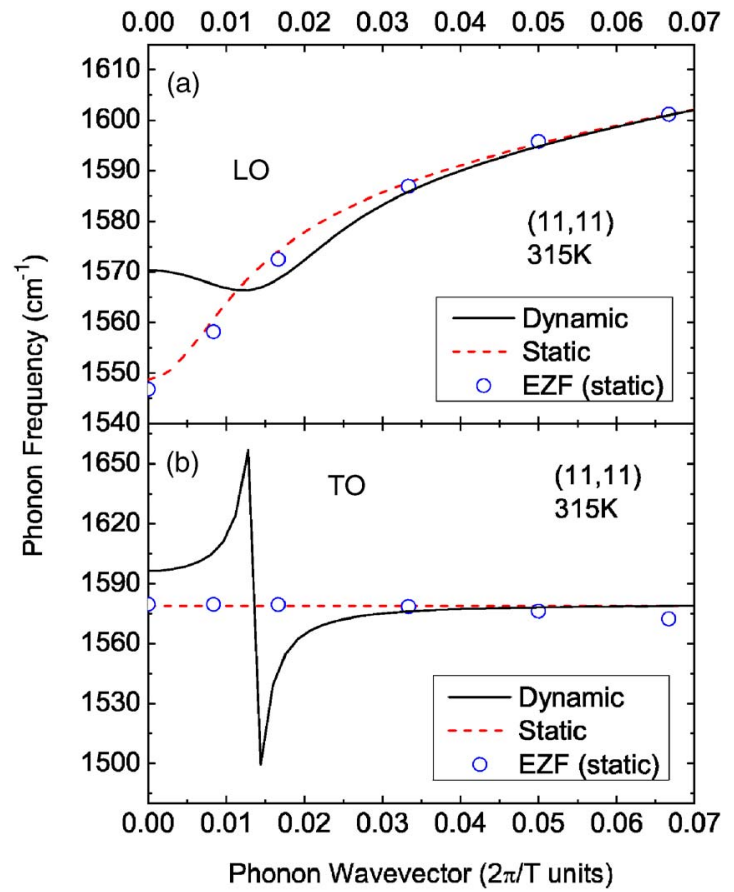

FIG. 12. (Color online) KA for the LO and TO branches of an $(11,11)$ tube close to $\boldsymbol{\Gamma}$. Phonon frequencies are calculated by means of static EZF-DFT (open dots), or from the numerical integration of the dynamical matrix in its static (dashed line) and dynamic (solid line) expression. $T_{e}=315 \mathrm{~K}$.

$2 \pi / a_{0}$ units, the static and dynamic results are indistinguishable.

It is important to observe that Eq. (27), which was derived within the static approximation, is also valid in the dynamic case. Indeed, for $T_{e} \rightarrow 0$, metallic SWNTs undergo a PD, meaning that the frequency of the phonon affected by the KA goes to zero. For $\hbar \omega_{\mathrm{LO}-\mathrm{TO}}=0$ the dynamic equations reduces to the static case, so $T_{\mathrm{PD}}$ is the same.

Figures 13 and 14 plot, respectively, the KA shape in the LO and TO branches as a function of diameter and $T_{e}$. The general trends observed within the static approximation are preserved also after the introduction of the dynamic effects. In particular, the LO phonon softening still increases for decreasing diameter and $T_{e}$. In addition, the phonon softening and/or hardening of the TO branch increases for decreasing diameters, but has no dependence on $T_{e}$.

\section{A. Dynamic effects in graphene and graphite}

In this section we have shown that dynamic effects induce huge modifications in the phonon dispersion of metallic nanotubes close to the Kohn anomaly. The demonstration of the importance of dynamic effects in one-dimensional systems opens the discussion on whether similar effects can be relevant in materials of higher dimensionality, graphite and graphene in particular. Indeed, it is possible to prove that dynamic effects can be crucial also for the correct description of the vibrational properties of bidimensional metallic systems. We have recently demonstrated, both theoretically and experimentally, that for electrically doped graphene the 


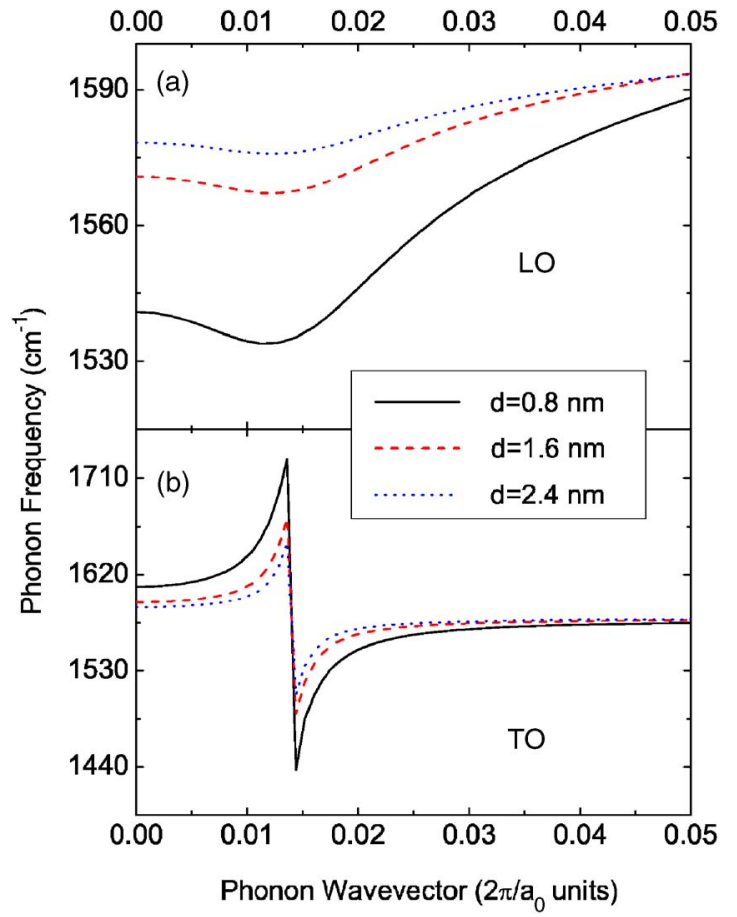

FIG. 13. (Color online) KA of the LO and TO branches for tubes of different diameters calculated at $T_{e}=315 \mathrm{~K}$ including dynamical effects.

Born-Oppenheimer approximation spectacularly fails. ${ }^{77-79}$ In particular the evolution of the $G$ peak position as a function of gate bias can be explained only by taking into account nonadiabatic effects, as will be reported in details elsewhere. ${ }^{78,79}$

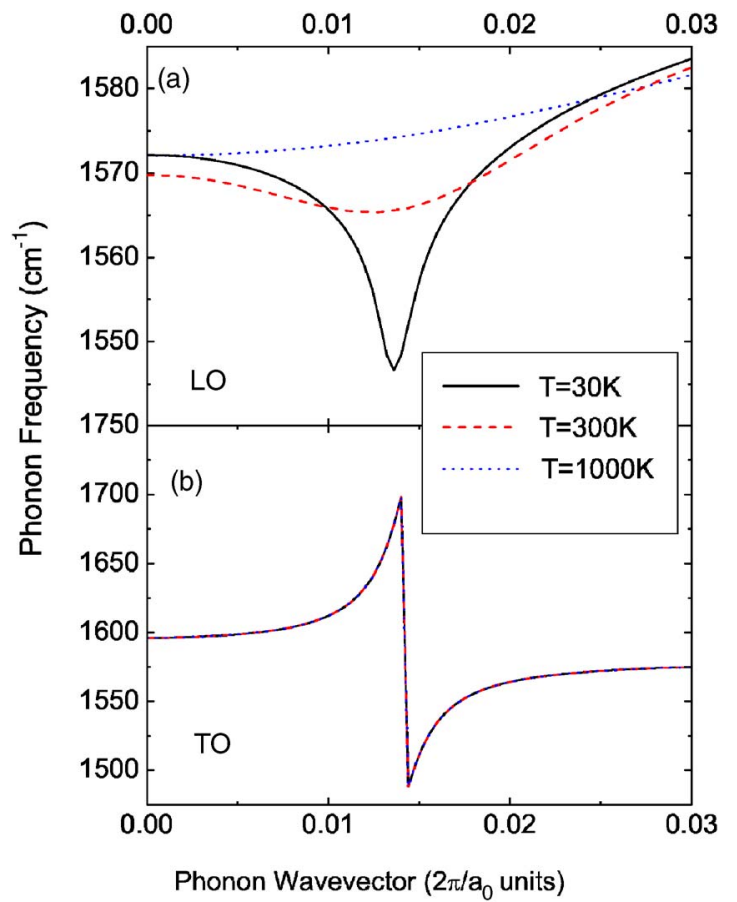

FIG. 14. (Color online) KA of LO and TO branches of tubes with $d=1.5 \mathrm{~nm}$ calculated for $T_{e}$ ranging from 30 to $1000 \mathrm{~K}$, including dynamic effects.

\section{CURVATURE EFFECTS}

Curvature effects encompass all the differences between graphene and SWNTs due to the geometrical distortion of the $\mathrm{C}-\mathrm{C}$ bonds in the tubes [Eq. (12)]. In common with quantum confinement, curvature also splits the double degenerate $\boldsymbol{\Gamma}$ $-E_{2 g}$ phonon of graphene into two distinct phonons in SWNTs. This split occurs because the different strengths of the chemical bonds along the tube axis and the tube circumference result in different phonon frequencies for modes polarized along these two directions. ${ }^{4,5}$ The folding of graphene to give the SWNT cylindrical shape results in (i) change in atoms separation (ii) loss of C-C bond planarity, (iii) mixing of $\sigma$ and $\pi$ states, giving a $s p^{2} / s p^{3}$ character to the chemical bonds.

When the purely $s p^{2}$ bonds of graphene are deformed, they assume a hybrid $s p^{2} / s p^{3}$ character. Since $s p^{2}$ bonds are stiffer than $s p^{3}$ bonds, mixing the orbitals results in bond softening. This mixing is proportional to curvature, so it is minimum for $\mathrm{C}-\mathrm{C}$ bonds parallel to the axis, and maximum for bonds oriented along the circumference. ${ }^{4,5}$ Since longitudinal modes deform $\mathrm{C}-\mathrm{C}$ bonds parallel to the axis, while transverse modes deform $\mathrm{C}-\mathrm{C}$ bonds along the circumference, one expects curvature to soften the modes polarized along the tube circumference more than those polarized along the axis. This softening should increase with the $s p^{2} / s p^{3}$ mixing, and, thus with the SWNT curvature.

In this section, we investigate the effects of pure curvature on the LO-TO splitting. In Eq. (12) the dynamical matrix of a SWNT $\left(\hat{\Theta}_{T}\right)$ is written as the sum of the dynamical matrix of graphene $\left(\hat{\Theta}_{G}\right)$ and two additional terms describing the effects of curvature $\left(\hat{\Theta}_{\text {curv }}\right)$ and confinement $\left(\hat{\Theta}_{\text {conf }}\right)$. EZFDFT describes the contributions deriving from graphene and from the confinement effects $\left(\hat{\Theta}_{T}^{\text {flat }}=\hat{\Theta}_{G}+\hat{\Theta}_{\text {conf }}\right)$, thus

$$
\hat{\Theta}_{\text {curv }}=\hat{\Theta}_{T}-\hat{\Theta}_{G}-\hat{\Theta}_{\text {conf }}=\hat{\Theta}_{T}-\hat{\Theta}_{T}^{\text {flat }} .
$$

According to Eq. (32), the effects of pure curvature on a given SWNT can be obtained as the difference between calculations performed on the actual SWNT and the results obtained by means of EZF-DFT.

The frequency dependence on the diameter for the modes derived from the graphene $\Gamma-E_{2 g}$ was calculated by several authors. ${ }^{17,18,27}$ As already pointed out in Sec. IV, previous calculations of the LO phonons in metallic SWNTs are either not converged with respect to the $k$-point sampling ${ }^{27}$ or use a fictitious electronic temperature ${ }^{17,18,75}$ instead of the real one, and thus do not properly describe the KA effects. However, for semiconducting SWNTs, KA are not present. Thus, the DFT results of Refs. 17 and 18 for LO and TO modes in semiconducting SWNTs are reliable, and can be used to estimate the curvature effects for $\mathrm{LO}$ and TO modes.

To verify the equivalence of the calculations of Refs. 17 and 18 with the present, the phonon dispersions of graphene of Ref. 18 are compared with those of Ref. 12 (which is obtained with the same method and code as the present calculations). Away from the KA, the only noticeable difference is a $16 \mathrm{~cm}^{-1}$ rigid upshift of the highest optical branches. Thus, the two calculations are equivalent (after a downshift 


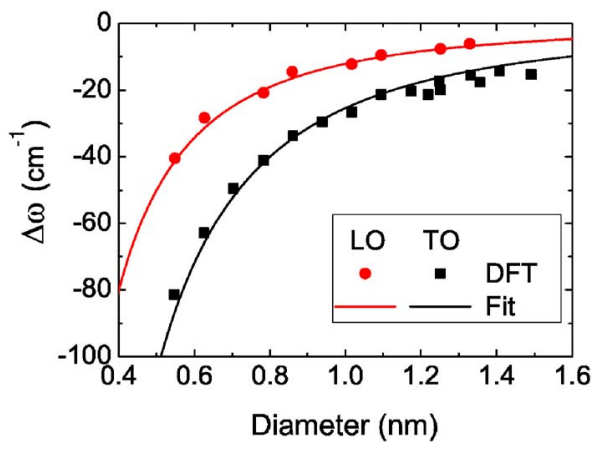

FIG. 15. (Color online) Curvature effects on LO and TO phonons. Dots represent the difference between the phonon frequencies of Ref. 18 and EZF-DFT of Sec. IV [Eq. (28))]. The lines are fit to the data done using Eq. (34).

of $16 \mathrm{~cm}^{-1}$ ) and is possible to extract the effects of pure curvature by comparing the results of Refs. 17 and 18 with the present EZF-DFT calculations. fine

Assuming the curvature effects as a perturbation, we de-

$$
\Delta \omega=\omega_{T}-\omega_{\text {flat }},
$$

where $\omega_{T}$ is the phonon frequency calculated on an actual tube and $\omega_{\text {flat }}$ is the EZF-DFT result. In Fig. 15 we plot $\Delta \omega$ as the difference between the phonon frequencies $\omega_{T}$ of Ref. 18 and the $\omega_{\text {flat }}$ from EZF-DFT as represented by Eq. (28). We consider only those modes which do not present any dependence on $T_{e}$. In particular, for the LO mode, we consider only semiconducting tubes, while for the TO mode, we consider both metallic and semiconducting tubes. For these modes, it is not necessary to distinguish between static and dynamic EZF-DFT. From a fit to the points of Fig. 15,

$$
\begin{aligned}
& \Delta \omega_{\mathrm{TO}}(d)=-\frac{\zeta_{\mathrm{TO}}}{d^{2}}, \\
& \Delta \omega_{\mathrm{LO}}(d)=-\frac{\zeta_{\mathrm{LO}}}{d^{2}},
\end{aligned}
$$

where $\zeta_{\mathrm{TO}}=25.16 \mathrm{~cm}^{-1} \mathrm{~nm}^{2}, \zeta_{\mathrm{LO}}=12.0 \mathrm{~cm}^{-1} \mathrm{~nm}^{2}$, and $d$ is the tube diameter in nm. We assume that Eq. (34) applies also to the LO mode of metallic tubes.

Figure 16 plots the diameter dependence of the LO-TO phonons as predicted considering only the effects of confinement (static and dynamic) or after the introduction of the curvature effects [added using Eq. (34)]. In metallic tubes, the relaxation of the static approximation introduces a diameter dependence for the TO phonon, and reduces the softening of the LO mode. This is a further confirmation that in SWNTs dynamic effects cannot be neglected. The most notable modification introduced by curvature is the strong diameter dependence of the TO mode in semiconducting tubes, which, according to pure confinement, was predicted to be diameter independent. Interesting, curvature almost perfectly compensates the effects of confinement for the TO mode in metallic tubes and for the LO mode in semiconducting tubes, resulting in almost diameter-independent phonons. Finally,

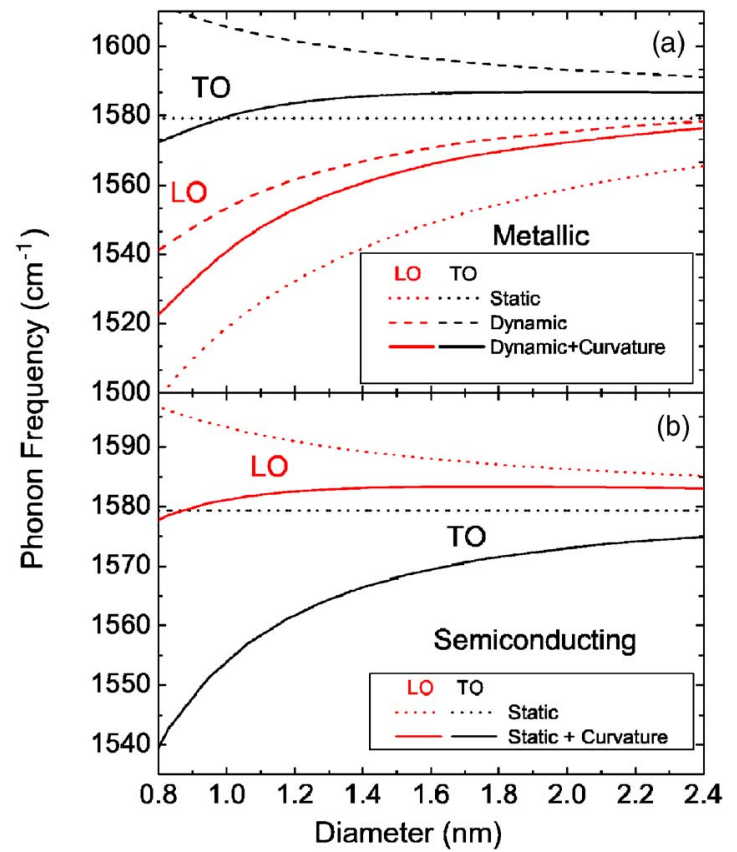

FIG. 16. (Color online) Comparison between LO and TO phonons. (a) for metallic tubes the phonon frequencies are calculated using EZF-DFT within the static approximation (dotted line), EZF-DFT including the dynamic effects (dashed line), and dynamic EZF-DFT correct with the curvature effects (solid lines). (b) for semiconducting tubes it is not necessary to distinguish between static and dynamic calculations; dotted lines represent the EZF-DFT calculations, solid lines represent EZF-DFT data corrected for the effects of curvature.

the effects of curvature in the LO modes of metallic tubes do not substantially modify the trend due to pure confinement, and result only in a correction of the phonon frequencies. Figure 16 is extremely important, and shows that curvature has the effect of inducing a noticeable phonon softening in small diameter SWNTs, for both LO and TO modes.

\section{A. LO-TO splitting}

In Secs. IV and V, we observed that quantum confinement has the effect of splitting the graphene $\Gamma-E_{2 g}$ phonon into a $\mathrm{LO}$ and a TO mode. Literature reports indicate that in chiral tubes curvature effects may induce deviations from this behavior. ${ }^{18,51}$ In Ref. 51, it was observed that the eigenvectors of the LO and TO phonons in the $(9,3)$ tube are aligned to the $\mathrm{C}-\mathrm{C}$ bonds, and deviate by $16^{\circ}$ from the axial and circumferential directions. However, in the same paper, it was also reported that for the $(8,4)$ tube the deviation is reduced to $2^{\circ}$. Calculations done in Ref. 18 show that for the $(12,6)$ tube the deviation from purely LO and TO eigenvectors is $4^{\circ}$. Using a symmetry adapted TB scheme, calculations performed in Ref. 31 on 300 different SWNTs indicated no substantial deviation of the atomic displacements from the axial and circumferential directions. Figure 17 shows the very different predictions given by our EZF-DFT and the calculations of Ref. 51.

The orientation of the atomic displacements in phonons derived from the $\boldsymbol{\Gamma}-E_{2 g}$ of graphene can be understood by 
(a)

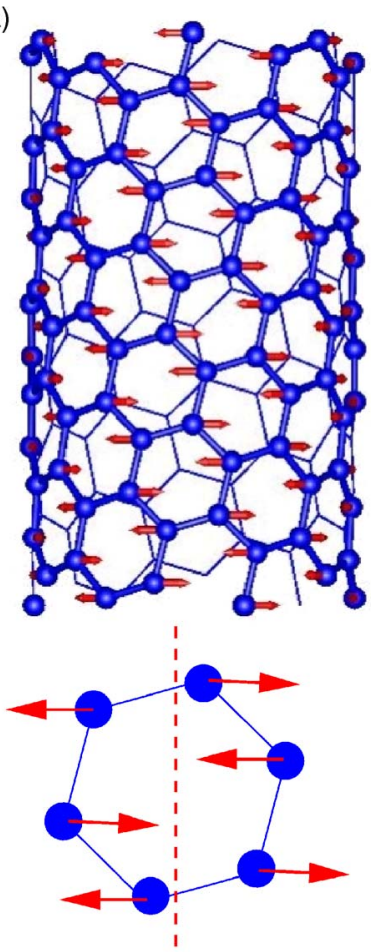

(b)

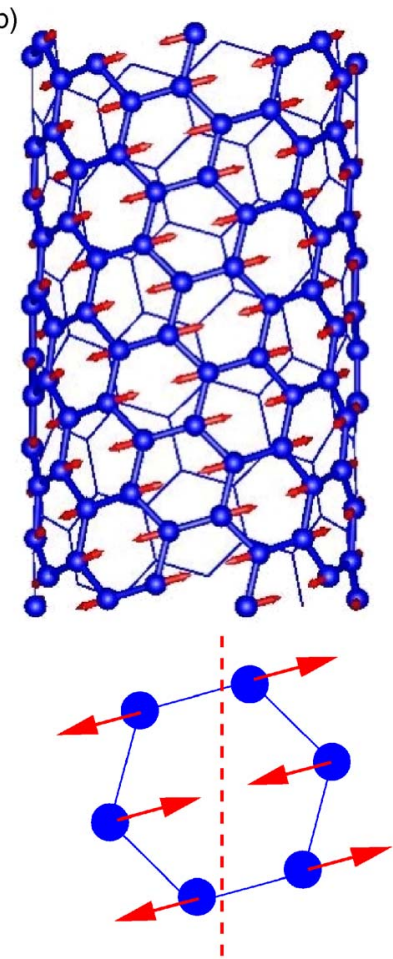

FIG. 17. (Color online) Pattern of atomic displacements associated to a phonon derived from the graphene $\boldsymbol{\Gamma}-E_{2 g}$ mode (a) as predicted by EZF-DFT and (b) as calculated in Ref. 51 .

considering the combined effects of confinement and curvature. The frequencies of the $\boldsymbol{\Gamma}-E_{2 g}$ modes of graphene are degenerate, and the corresponding eigenvectors can be oriented along any couple of in-plane directions. Curvature and confinement both have the effect of removing the degeneracy. The effect that curvature and confinement have in orienting the atomic displacements is proportional to the effect they have in determining the LO-TO splitting. These contributions can be read from Fig. 16, by comparing the curves before and after the introduction of the curvature corrections. In metallic tubes the effects of confinement are dominant over curvature, thus the modes derived from graphene $\boldsymbol{\Gamma}-E_{2 g}$ are expected to be almost perfectly LO and TO. In Sec. IV, and in Table III we pointed out that the calculations in Ref. 51 were done using a very limited number of $k$ points, and thus failed to describe the effects of confinement. This is also supported by the results presented in Ref. 18 . Indeed, calculations of Ref. 18 are converged with respect to the number of $k$ points, and show that for the $(12,6)$ tube the modes are almost perfectly LO and TO.

For semiconducting tubes, the effects of curvature are dominant over the effects of confinement. In this case, the almost perfect LO and TO character imposed by confinement can be substantially perturbed by curvature. However, it is important to remember that the splitting caused by curvature is due to the different force constants along the tubes axis and circumference. As a consequence, phonons that present a strong curvature induced splitting must be oriented along directions for which the difference between the force constants are significantly strong. This means that the atomic

displacements have to be substantially aligned along the tube axis and the tube circumference. This suggests that the deviations from purely axial and circumferential displacement have to be modest, and that it should be always possible to classify the modes as almost LO and almost TO.

\section{INTERPRETATION OF THE RAMAN $G$ BAND IN NANOTUBES}

We now show that combining curvature, confinement and dynamic effects we can interpret the SWNT $G$ peak.

The Raman spectrum of SWNT shows strong features in the $1540-1600 \mathrm{~cm}^{-1}$ spectral region. ${ }^{4-6,49,80-82}$ This is the same region of the $G$ peak in graphene, ${ }^{16}$ graphite, ${ }^{83}$ and amorphous and nanostructured carbons. ${ }^{84,85}$

As shown in Fig. 18, the $G$ band of SWNTs consists of two main peaks: $G^{+}$and $G^{-} .{ }^{86}$ In semiconducting tubes both peaks are sharp, while in metallic tubes the $G^{-}$is broader and downshifted. ${ }^{5,6,86-96}$ For both metallic and semiconducting tubes, the position of the $G^{-}$peak depends on the tube diameter, having lower frequency for smaller diameters, while the position of the $G^{+}$peak is substantially diameter independent. ${ }^{86}$ As discussed in Sec. VI, curvature affects more strongly the circumferential modes, thus the $G^{+}$and $G^{-}$ are commonly assigned to LO (axial) and TO (circumferential) modes, respectively. ${ }^{5,86-88,90,91}$

Conflicting reports exist on the presence and relative intensity of the $G^{-}$band in isolated versus bundled metallic tubes. Some groups report that the intensity of this peak in isolated tubes is the same as in bundles, ${ }^{86,95,96}$ while others observe that it is smaller ${ }^{91,93,94}$ or even absent. ${ }^{97}$ The downshift and the broadening of the $G^{-}$peak in metallic tubes is commonly attributed to the onset of a Fano resonance between plasmons and the TO phonon..$^{90-92,97,98}$ Such phononplasmon coupling would either need ${ }^{92}$ or not need ${ }^{90,98}$ a finite phonon wave vector for its activation. The theory of Refs. 90 and 98 predicts the phonon-plasmon peak to be intrinsic in single SWNT, in contrast with Ref. 97. On the other hand, the theory in Ref. 92 requires several tubes $(>20)$ in a bundle in order to observe a significant $G^{-}$intensity, in contrast with the experimental observation that bundles with very few metallic tubes show a significant $G^{-} \cdot{ }^{93-97}$ Reference 92 also predicts a $G^{-}$upshift with number of tubes in the bundle, in contrast with Ref. 97, which shows a downshift, and with Refs. 86,90 , and 95 , which show that the $G^{-}$position depends on the tube diameter and not bundle size. Finally, the $G^{-}$position predicted by Refs. 92 and 98 is at least $200 \mathrm{~cm}^{-1}$ lower than that measured. $5,6,86-91,93-96$

Thus, all the proposed theories for phonon-plasmon coupling $90,92,98$ are qualitative, require the guess of several quantities, and fail to predict in a precise, quantitative, parameter-free way the observed line shapes and their diameter dependence.

Phonon symmetry proves that there are only six Ramanactive modes at $\Gamma:{ }^{48,80,82,100}$ two $A$, two $E_{1}$, and two $E_{2}$. The $A$ modes are totally symmetric, have the highest EPC, and correspond to the LO and TO modes we examined in the preceding sections. In Fig. 19 we compare the experimentally measured $G^{+}$and $G^{-}$peaks to the calculated frequencies 


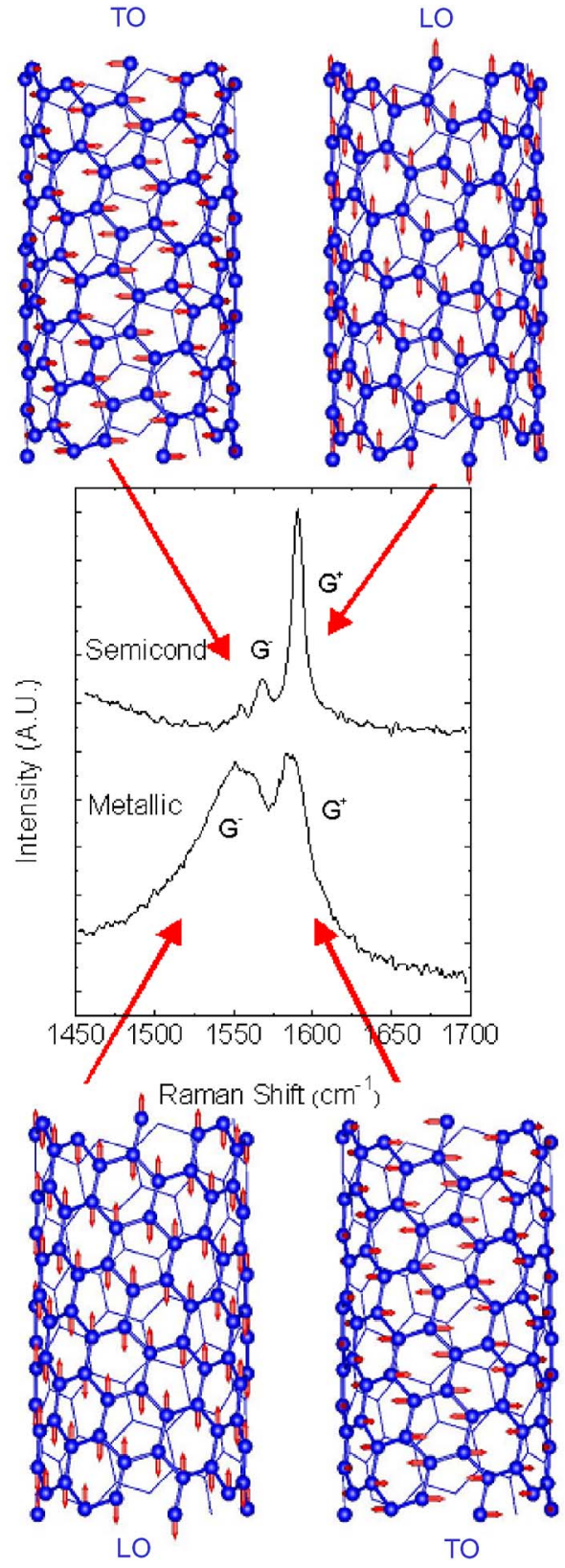

FIG. 18. (Color online) Raman $G^{+}$and $G^{-}$peaks for semiconducting and metallic SWNTs (spectra adapted from Ref. 82). In semiconducting tubes the two peaks have a Lorentzian shape and a FWHM of $\sim 12 \mathrm{~cm}^{-1}$. In metallic tubes the $G^{-}$peak is downshifted and much broader. Our assignment of the spectral features is also indicated.

of the LO and TO phonons. The plot of the phonon frequencies corresponds to the results of static and dynamic EZFDFT plus the curvature correction $\Delta \omega$.

For semiconducting tubes, our calculations support the $G^{+}, G^{-}$assignment to the $\mathrm{LO}$ and TO phonons.

For metallic tubes, our LO and TO positions reproduce the diameter dependence of the $G^{-}$and the $G^{+}$peaks, respectively. Our assignment of the $G$ peaks in metallic tubes is thus the opposite of that in semiconducting. This is emphasized in Fig. 18, where we represent the correspondence between the Raman peaks and the LO-TO phonons.

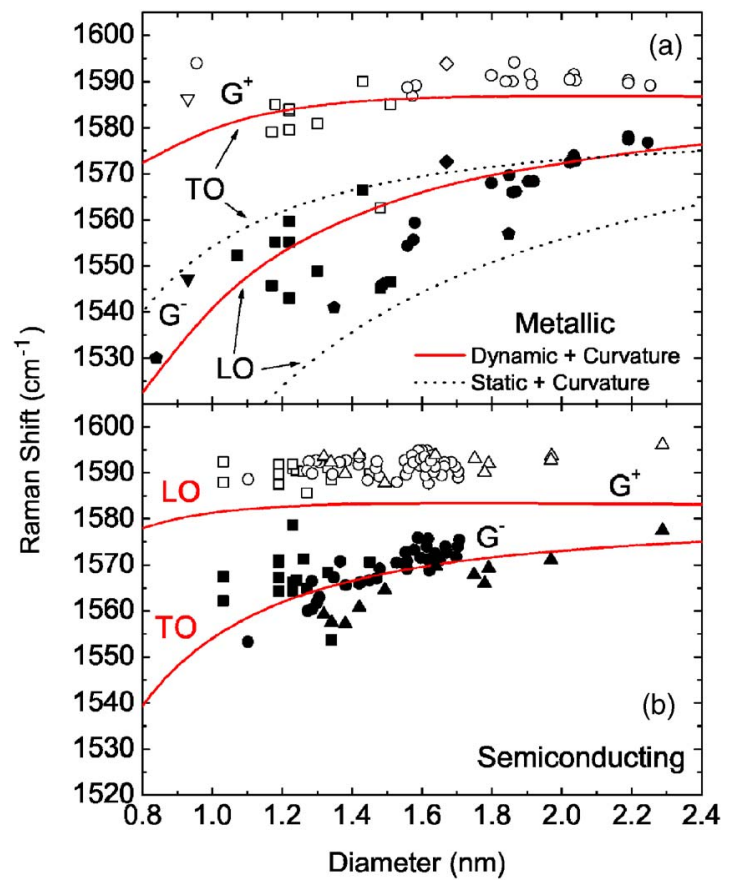

FIG. 19. (Color online) Comparison between LO and TO modes of (a) metallic and (b) semiconducting tubes and the experimental position of the $G^{+}$and $G^{-}$peaks of the Raman spectra of SWNTs. The position of the LO and TO modes is calculated using EZF-DFT and adding the curvature correction. In panel (a) continuous lines represent the results of dynamic EZF-DFT calculations, while dotted lines refer to static EZF. Open and filled symbols represent, respectively, the measured position of the $G^{+}$and $G^{-}$peaks. Data from Oron et al. (Ref. 95) (squares), Jorio et al. (Ref. 86) (circles), Maultzsch et al. (Ref. 93), (triangles), Doorn et al. (Ref. 96) (diamonds), Meyier et al. (Ref. 99) (hexagons), and Brown et al. (Ref. 90) (pentagons).

Our calculations prove that the $G^{-}$softening can be precisely predicted by simply considering the coupling of the LO phonon with single-particle electronic excitations.

Furthermore, in Ref. 70, we have shown that our DFT calculated EPC also account for the measured FWHM. Vice versa the experimental FWHM can be used to measure the EPC. ${ }^{70}$ It is important to remind here that only single resonance Raman can detect a $G^{-}$with large FWHM in metallic nanotubes. ${ }^{70}$ The same metallic nanotubes measured in double resonance would have a sharp $G^{-} .70,93$

It is also interesting to consider if Raman scattering can allow to probe regions very close to the anomalies in Figs. $12-14$. This is not possible in the single resonance approach, since it only probes $\mathbf{q}=0$. This might be possible in double resonant Raman scattering. In this case the $\mathbf{q}=0$ rule is relaxed, and the scattering process involves phonons with $\mathbf{q}$ $\neq 0 .{ }^{49,101}$ However, only phonons with $q>\hbar \omega_{\Gamma} / \beta$ satisfy the conservation of energy and momentum in a double resonant process. Figure 12 shows that for such $q$ the anomaly is missed and the predicted peak position is very similar to the $q=0$ case. However, crossing from single to double resonant would result in a significant downshift of the TO mode, as for Eq. (31). This is consistent with the experimental data presented in Ref. 93. 


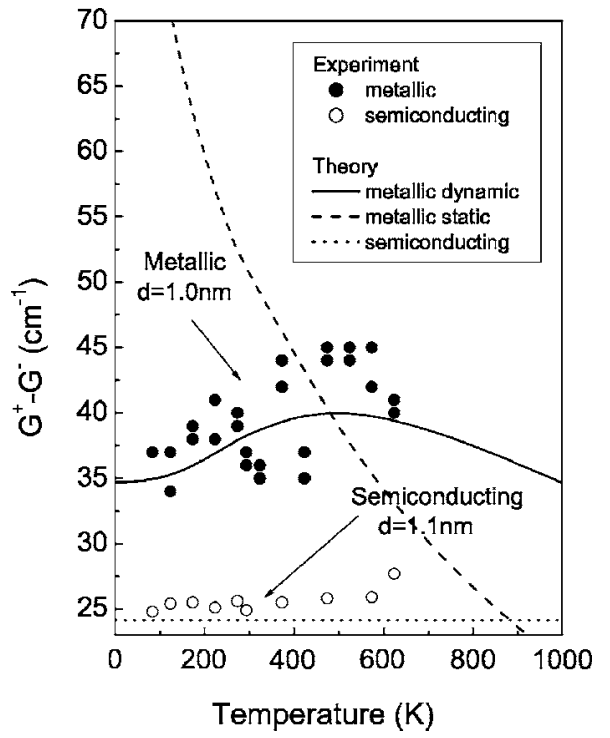

FIG. 20. $T_{e}$ dependence of LO-TO splitting for a metallic and a semiconducting tube, calculated using EZF-DFT and including curvature corrections. The solid line corresponds to results of dynamic calculations on a metallic tube; the dashed line corresponds to static calculations. The splitting for a semiconducting tube is represented by the dotted line.

Further validation of our calculations with experiments is provided by the analysis of the temperature dependence of the $G^{+}$and $G^{-}$positions.

As stressed in the preceding sections, here we do not describe the frequency shift induced by the presence of anharmonic effects. However, the effects of anharmonicity are expected to be the same for the LO and TO phonons. Thus anharmonicity does not affect the relative position of these two phonons, which can then be altered only by effects related to $T_{e}$. The temperature dependence of the $G^{+}-G^{-}$splitting can be investigated using our analytical model.

The difference between the frequency of the LO and TO modes for metallic and semiconducting tubes of similar diameter $d=1 \mathrm{~nm}$ is plotted as a function of $T_{e}$ in Fig. 20. For the semiconducting tube no effects related to $T_{e}$ are expected, thus the splitting is temperature independent. For metallic tubes, the static and the dynamic model give very different predictions. According to the static model, the frequency split should have a strong logarithmic dependence on temperature, Eq. (26). On the contrary the dynamic model, obtained by the numerical integration of Eq. (10), predicts that the splitting (i) has a maximum at $T_{e} \sim 500 \mathrm{~K}$, (ii) it saturates for $T_{e} \rightarrow 0$, (iii) for $T_{e} \rightarrow \infty$ it decrease monotonically, and (iv) in the range $T=0 \rightarrow 1000 \mathrm{~K}$ it varies less than $10 \mathrm{~cm}^{-1}$. This variation is function of the tube diameter, and is plotted in Fig. 21. We observe that this value is extremely small $\left(\sim 2 \mathrm{~cm}^{-1}\right)$ for large diameters, but rapidly increases for $d<1.5 \mathrm{~nm}$.

Experimental data from Ref. 77, obtained from tubes having $d \sim 1.0 \mathrm{~nm}$, are in excellent agreement with the prediction of the dynamical model. This confirms the validity of our model and the inadequacy of a static approach for the investigation of the vibrational properties of SWNTs.

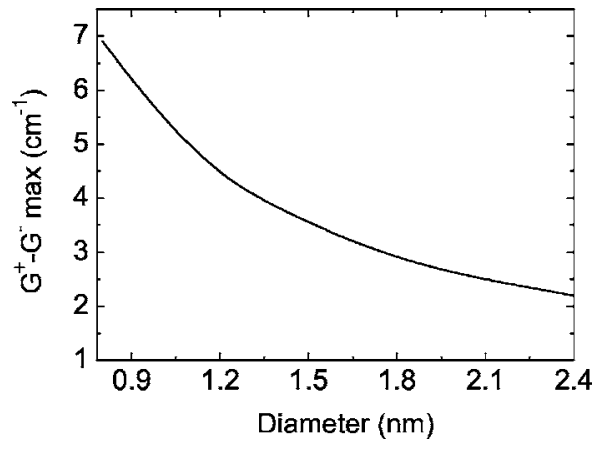

FIG. 21. Difference between the saturation value at $T_{e}=0 \mathrm{~K}$ and the maximum value at $T_{e} \sim 500 \mathrm{~K}$ of the frequency difference of the $G^{+}$and the $G^{-}$Raman peaks as a function of the tubes diameter. The values are calculated using dynamic EZF-DFT and including curvature corrections.

\section{CONCLUSIONS}

We presented a detailed theoretical investigation of the optical phonons of SWNTs. We first analyzed the static DFPT approach and singled out the effects of curvature and confinement. Confinement plays a major role in shaping SWNT phonons and is often more relevant than curvature. We presented an electronic zone folding method allowing the evaluation of confinement effects on phonon-dispersions and electron-phonon coupling of SWNTs of any diameter and chirality, and for any electronic temperature. We investigated Kohn anomalies and Peierls distortions in metallic SWNTs with diameters up to $3 \mathrm{~nm}$ and in a $T_{\mathrm{e}}$ range from 4 to $3000 \mathrm{~K}$. We then presented a simple analytic model exactly accounting for all the static DFPT results. Finally, we proved that non adiabatic dynamic effects, beyond the BornOppenheimer approximation, induce significant changes in the occurrence and shape of Kohn anomalies. We have shown that it is necessary to consider dynamic effects in order to correctly describe the phonon dispersions of SWNTs, in contrast to what happens in most other materials. Finally we discussed the interpretation of the Raman $G$ peak in SWNTs. Only by combining dynamic, curvature and confinement effects we can reach an agreement between experiments and theory. In metallic SWNTs, we assign the $G^{+}$and $G^{-}$peaks to the TO (circumferential) and LO (axial) mode, the opposite of semiconducting SWNTs.

\section{ACKNOWLEDGMENTS}

Calculations were performed at IDRIS (Orsay, Grant No. 051202) and HPCF (Cambridge). One of the authors (S.P.) acknowledges funding from Pembroke College, Cambridge, the EU Marie Curie Program IHP-HPMT-CT-2000-00209 and EU project CANAPE. One of the authors (A.C.F.) acknowledges support from EPSRC Grant No. GR/S97613, the Royal Society and the Leverhulme Trust.

\section{APPENDIX A}

Here we derive the relation between the SWNT and graphene EPC presented in Sec. III, Eq. (13). 
By definition of the EPC [Eq. (5)],

$$
D_{(\mathbf{k}+\mathbf{q}) n, \mathbf{k} m}=\left\langle\psi_{\mathbf{k}+\mathbf{q}, n}\left|\frac{\partial \hat{H}}{\partial \mathbf{R}} \boldsymbol{\varepsilon}_{\mathbf{q}, \eta}\right| \psi_{\mathbf{k}, m}\right\rangle,
$$

where $\hat{H}$ is the system Hamiltonian, $\mathbf{R}$ represents the atomic coordinates, $\left|\psi_{\mathbf{k}, n}\right\rangle$ is the electronic state of wave vector $\mathbf{k}$ and band $n$, and $\boldsymbol{\varepsilon}_{\mathbf{q}, \eta}$ is the polarization of a phonon of wave vector $\mathbf{q}$ and branch $\eta$. We define $\left|\psi_{\mathbf{k}, n}^{1}\right\rangle$ and $\boldsymbol{\varepsilon}_{\mathbf{q}, \eta}^{1}$ the wave function and polarization normalized to 1 on the unit cell. We also define $\left|\psi_{\mathbf{k}, n}^{N}\right\rangle$ and $\boldsymbol{\varepsilon}_{\mathbf{q}, \eta}^{N}$ the wave functions and the polarizations normalized to 1 on a supercell composed by $N$ unit cells,

$$
\begin{aligned}
\left|\psi_{\mathbf{k}, n}^{N}\right\rangle & =\frac{1}{\sqrt{N}}\left|\psi_{\mathbf{k}, n}^{1}\right\rangle, \\
\boldsymbol{\varepsilon}_{\mathbf{q}, \eta}^{N} & =\frac{1}{\sqrt{N}} \boldsymbol{\varepsilon}_{\mathbf{q}, \eta}^{1} .
\end{aligned}
$$

The EPC calculated on the supercell is then

$$
D_{(\mathbf{k}+\mathbf{q}) n, \mathbf{k}, m}^{N}=\left\langle\psi_{\mathbf{k}+\mathbf{q}, n}^{N}\left|\frac{\partial \hat{H}}{\partial \mathbf{R}} \boldsymbol{\varepsilon}_{\mathbf{q}, \eta}^{N}\right| \psi_{\mathbf{k}, m}^{N}\right\rangle .
$$

This integral is equivalent to the sum of $N$ unit cell integrals:

$$
D_{(\mathbf{k}+\mathbf{q}) n, \mathbf{k}, m}^{N}=N\left\langle\frac{\psi_{\mathbf{k}+\mathbf{q}, n}^{1}}{\sqrt{N}}\left|\frac{\partial \hat{H}}{\partial \mathbf{R}} \frac{\boldsymbol{\varepsilon}_{\mathbf{q}, \eta}^{1}}{\sqrt{N}}\right| \frac{\psi_{\mathbf{k}, m}^{1}}{\sqrt{N}}\right\rangle=\frac{1}{\sqrt{N}} D_{(\mathbf{k}+\mathbf{q}) n, \mathbf{k}, m}^{1} .
$$

Considering a SWNT with a unit-cell containing $N$ graphene unit cells, Eq. (13) is immediately obtained.

Equation (A3) states that the EPC depends on the supercell choice, as a consequence the EPC per se is not a physical observable. In general, a physical observable is given by the product of the square of the EPC times an electronic density of states $\rho$,

$$
\rho(\epsilon)=\frac{1}{N_{\mathbf{k}}} \sum_{\mathbf{k} n} \delta\left[\epsilon-\epsilon_{n}(\mathbf{k})\right],
$$

where the sum is performed on $N_{\mathbf{k}}$ wave vectors $\mathbf{k}$ and on the bands $n . \epsilon_{n}(\mathbf{k})$ is the energy of $\left|\psi_{\mathbf{k}, n}\right\rangle$. One can easily check that $\rho$ is proportional to $N$, the size of the supercell. Thus, the product $|D|^{2} \rho$ does not depend on the supercell chosen and can correspond to a physical observable.

\section{APPENDIX B}

We evaluate Eq. (16) to obtain the shape of the KA at $T$ $=0$, presented in Sec. IV E. For $T=0 \mathrm{~K}$, the occupation factors $f$ in Eq. (16) are 0 or 1 . For $\mathbf{q} \sim \boldsymbol{\Gamma}$ the nonanalytic component of the dynamical matrix is then

$$
\widetilde{\Theta}_{\mathbf{q} \sim \Gamma}=\frac{A_{\Gamma} \sqrt{3} a_{0}^{2}}{\pi^{2} d} \int_{-\bar{k}}^{\bar{k}} \frac{\left|\widetilde{D}_{\left(\mathbf{K}+\mathbf{k}^{\prime}+\mathbf{q}\right) \pi^{*},\left(\mathbf{K}+\mathbf{k}^{\prime}\right) \pi}\right|^{2}}{\epsilon_{\left(\mathbf{K}+\mathbf{k}^{\prime}\right) \pi}-\epsilon_{\left(\mathbf{K}+\mathbf{k}^{\prime}+\mathbf{q}\right) \pi^{*}}} d k^{\prime}
$$

where $\pi$ and $\pi^{*}$ indicate bands occupied or empty, respectively (Fig. 2). Neglecting the dependence of $|\widetilde{D}|$ on $k^{\prime}$ and assuming a linear dispersion for the electronic bands $\epsilon_{\left(\mathbf{K}+\mathbf{k}^{\prime}\right) \pi}=-\beta k^{\prime}$ and $\epsilon_{\left(\mathbf{K}+\mathbf{k}^{\prime}\right) \pi^{*}}=+\beta k^{\prime}$, Eq. (B1) becomes

$$
\begin{aligned}
\tilde{\Theta}_{\mathbf{q} \sim \Gamma}= & \frac{A_{\boldsymbol{\Gamma}} \sqrt{3} a_{0}^{2}}{\pi^{2} d}\left(\left|\tilde{D}_{L R}\right|^{2} \int_{-\bar{k}}^{-q} \frac{1}{\beta\left(2 k^{\prime}+q\right)} d k^{\prime}\right. \\
& +\left|\tilde{D}_{L L}\right|^{2} \int_{-q}^{0} \frac{1}{-\beta q} d k^{\prime} \\
& \left.+\left|\tilde{D}_{R L}\right|^{2} \int_{0}^{+\bar{k}} \frac{1}{-\beta\left(2 k^{\prime}+q\right)} d k^{\prime}\right) .
\end{aligned}
$$

Here, the subscripts of $D$ indicate whether we are considering electronic bands $L$ or $R$ (Fig. 2). The integrals can be easily evaluated. Considering $\ln |2 \bar{k}+q| \sim \ln |2 \bar{k}|=C_{\bar{k}}^{-}$and $\left|\widetilde{D}_{L R}\right|^{2}=\left|\widetilde{D}_{R L}\right|^{2}$, we have

$$
\widetilde{\Theta}_{\mathbf{q} \sim \Gamma}=\frac{A_{\Gamma} \sqrt{3} a_{0}^{2}}{\pi^{2} d \beta}\left[\left|\tilde{D}_{L R}\right|^{2}\left(\ln |q|-C_{\bar{k}}^{-}\right)-\left|\tilde{D}_{L L}\right|^{2}\right] .
$$

We now distinguish between the two LO and TO branches at $\mathbf{q} \sim \boldsymbol{\Gamma}$. From Table I, we get $\left|\widetilde{D}_{L R}\right|=2\left\langle D_{\Gamma}^{2}\right\rangle_{F}$ for the LO branch and 0 for the TO, while $\left|\widetilde{D}_{L L}\right|=2\left\langle D_{\Gamma}^{2}\right\rangle_{F}$ for the TO and 0 for the LO, thus

$$
\begin{gathered}
\widetilde{\Theta}_{\mathrm{LO}}=\frac{A_{\Gamma} \sqrt{3} a_{0}^{2} 2\left\langle D_{\Gamma}^{2}\right\rangle_{F}}{\pi^{2} d \beta} \ln |q|+C_{\bar{k}}^{\prime}, \\
\tilde{\Theta}_{\mathrm{TO}}=-\frac{A_{\Gamma} \sqrt{3} a_{0}^{2} 2\left\langle D_{\Gamma}^{2}\right\rangle_{F}}{\pi^{2} d \beta},
\end{gathered}
$$

where $\widetilde{\Theta}_{\mathrm{LO}}\left(\widetilde{\Theta}_{\mathrm{TO}}\right)$ is the nonanalytic component of the dynamical matrix related to the LO (TO) branch. $C_{\bar{k}}^{\prime}$ $=C_{k}^{-} A_{\Gamma} \sqrt{3} a_{0}^{2} 2\left\langle D_{\Gamma}^{2}\right\rangle_{F} / \pi^{2} d \beta$ contains all $\bar{k}$ dependent terms. For $q \rightarrow 0 \widetilde{\Theta}_{\mathrm{LO}} \rightarrow-\infty$, while $\widetilde{\Theta}_{\mathrm{TO}}$ is a constant independent on $q$. This explains the occurrence of the KA only for the LO branch and its absence for the TO branch in static DFPT.

The phonon frequencies $\omega$ are obtained by $\omega^{2}=\operatorname{Re}\{(\widetilde{\Theta}$ $\left.\left.+\Theta^{\text {an }}\right) / M\right\}$, where $M$ is the carbon mass and the analytic component of the dynamical matrix $\Theta^{\text {an }}$ is expected to have a very weak dependence on $\mathbf{q}$. Concluding, at $T=0$, for $\mathbf{q}$ $\sim \boldsymbol{\Gamma}$, the frequencies of the LO and TO branches are

$$
\omega_{\mathrm{LO}}^{2}=\frac{A_{\Gamma} \sqrt{3} a_{0}^{2} 2\left\langle D_{\Gamma}^{2}\right\rangle_{F}}{\pi^{2} d \beta M} \ln |q|+C_{\mathrm{LO}}=\frac{\alpha_{\Gamma}}{d} \ln |q|+C_{\mathrm{LO}}
$$

$$
\omega_{\mathrm{TO}}^{2}=-\frac{A_{\Gamma} \sqrt{3} a_{0}^{2} 2\left\langle D_{\Gamma}^{2}\right\rangle_{F}}{\pi^{2} d \beta M}+C_{\mathrm{TO}}=-\frac{\alpha_{\Gamma}}{d}+C_{\mathrm{TO}},
$$

where the constants $C_{\mathrm{LO}-\mathrm{TO}}$ include all the contributions from the analytic part of the dynamical matrix $\Theta^{\text {an }}$ and all the nondivergent terms of $\widetilde{\Theta}$. For the numerical evaluation of $\alpha_{\Gamma}$ we use $A_{\Gamma}=2, \quad a_{0}=2.46 \AA, \quad \beta=5.52 \mathrm{eV} \AA, \quad\left\langle D_{\Gamma}^{2}\right\rangle_{F}$ $=45.60 \mathrm{eV}^{2} \AA^{-2}, M=12.01$ a.m.u. The corresponding equations for the KA at $\mathbf{K}$ are obtained in a completely analogous way. 


\section{APPENDIX C}

We determine the dependence on $T_{e}$ of the KA phonon frequencies at $\mathbf{q}=\boldsymbol{\Gamma}$ and $\mathbf{q}=\mathbf{K}$. We define

$$
f(x)=\frac{\theta(x)-\theta(-x)}{x} .
$$

Using Eq. (21), Eq. (25) becomes

$$
\tilde{\Theta}_{\Gamma / \mathbf{K}}=\frac{\alpha_{\Gamma / \mathbf{K}}}{d} \int_{0}^{\bar{x}} f(x) d x,
$$

where $\bar{x}=\beta \bar{k} /\left(T k_{B}\right)$. We observe that $\lim _{x \rightarrow 0} f(x)=0$ and that for $x \rightarrow \infty f(x) \sim-\frac{1}{x}$. Since $\bar{k}$ is chosen in the linear range of the $\pi$ and $\pi^{*}$ bands, $\beta k^{\prime}$ is a fraction of eV. Moreover, $k_{B}$ $=8.617 \times 10^{-5} \mathrm{eV} / \mathrm{K}$, so the condition $\beta k^{\prime} \gg k_{B} T$, corresponding to the $\bar{x} \rightarrow \infty$, is reached for $T$ up to thousands $\mathrm{K}$, well above any realistic experimental condition. In Eq. (C2) we replace $f(x)$ with

$$
\begin{aligned}
& \tilde{f}(x)=f(x) \quad \text { for } x<x_{1}, \\
& \widetilde{f}(x)=-\frac{1}{x} \quad \text { for } x>x_{1} .
\end{aligned}
$$

Equation (C2) becomes

$$
\tilde{\Theta}_{\Gamma / \mathbf{K}}=\frac{\alpha_{\Gamma / \mathbf{K}}}{d}\left(\int_{0}^{x_{1}} f(x) d x-\int_{x_{1}}^{\bar{x}} \frac{1}{x} d x\right) .
$$

Defining $F(x)=\int f(x) d x$, we obtain

$$
\begin{aligned}
\tilde{\Theta}_{\Gamma / \mathbf{K}} & =\frac{\alpha_{\Gamma / \mathbf{K}}}{d}\left[F\left(x_{1}\right)-F(0)-\ln \bar{x}+\ln x_{1}\right] \\
& =\frac{\alpha_{\Gamma / \mathbf{K}}}{d} \ln \frac{x_{0}}{\bar{x}} \\
& =\frac{\alpha_{\Gamma / \mathbf{K}}}{d} \ln \frac{T}{\bar{T}_{0}},
\end{aligned}
$$

where $\ln \left(x_{0}\right)=F\left(x_{1}\right)-F(0)+\ln \left(x_{1}\right)$ and $T_{0}=\frac{\beta \bar{k}}{k_{B} x_{0}}$. Considering that the analytic part of the dynamical matrix [Eq. (19)] as independent from $T$, Eq. (26) is then obtained.

\section{APPENDIX D}

We evaluate the frequency upshift of the TO mode due to dynamic effects. From Eq. (30), we observe that, for $q=0$, $\widetilde{D}_{\mathrm{TO}}=0$. Thus, from Eq. (19), one obtains that for $q=0$,

$$
\left(\omega_{\mathrm{TO}}^{\mathrm{dyn}}\right)^{2}=\frac{\Theta_{\mathrm{TO}}^{\mathrm{an}}}{M} .
$$

Since the contribution of the phonon energy in the denominator of $\Theta_{\mathbf{q}}$ is negligible for $\mathbf{k}$ away from the Fermi surface, $\Theta_{\mathbf{q}}^{\mathrm{an}}$ is the same in the static and in the dynamic case. Using Eqs. (19)-(21), we obtain

$$
\frac{\Theta_{\mathrm{TO}}^{\mathrm{an}}}{M}=\left(\omega_{\mathrm{TO}}^{\text {stat }}\right)^{2}-\frac{\alpha_{\Gamma}}{d} .
$$

Eqs. (D1) and (D2) gives

$$
\left(\omega_{\mathrm{TO}}^{\mathrm{dyn}}\right)=\sqrt{\left(\omega_{\mathrm{TO}}^{\text {stat }}\right)^{2}-\frac{\alpha_{\Gamma}}{d}} .
$$

Equation (31) is finally obtained by using $\sqrt{1+x} \simeq 1+x / 2$ and substituting the numerical value of $\alpha_{\Gamma}$.
*Electronic address: acf26@eng.cam.ac.uk

'Electronic address: mauri@lmcp.jussieu.fr

${ }^{1}$ A. Oberlin, M. Endo, and T. Koyama, J. Cryst. Growth 32, 335 (1976).

${ }^{2}$ S. Iijima, Nature (London) 354, 56 (1991).

${ }^{3}$ A. M. Rao, E. Richter, S. Bandow, B. Chase, P. C. Eklund, K. A. Williams, S. Fang, K. R. Subbaswamy, M. Menon, A. Thess et al., Science 275, 187 (1997).

${ }^{4}$ R. Saito, G. Dresselhaus, and M. S. Dresselhaus, Physical Properties of Carbon Nanotubes (Imperial College Press, London, 1998).

${ }^{5}$ S. Reich, C. Thomsen, and J. Maultzsch, Carbon Nanotubes: Basic Concepts and Physical Properties (Wiley-VCH, Weinheim, UK, 2004).

${ }^{6}$ A. C. Ferrari and J. Robertson, Philos. Trans. R. Soc. London, Ser. A 362, 2267 (2004).

${ }^{7}$ Z. Yao, C. L. Kane, and C. Dekker, Phys. Rev. Lett. 84, 2941 (2000)

${ }^{8}$ J. Y. Park, S. Rosenblatt, Y. Yaish, V. Sazonova, H. Ustunel, S. Braig, T. A. Arias, P. W. Brouwer, and P. L. McEuen, Nano Lett. 4, 517 (2004).
${ }^{9}$ A. Javey, J. Guo, M. Paulsson, Q. Wang, D. Mann, M. Lundstrom, and H. Dai, Phys. Rev. Lett. 92, 106804 (2004).

${ }^{10}$ M. Lazzeri, S. Piscanec, F. Mauri, A. C. Ferrari, and J. Robertson, Phys. Rev. Lett. 95, 236802 (2005).

${ }^{11}$ M. Lazzeri and F. Mauri, Phys. Rev. B 73, 165419 (2006).

${ }^{12}$ S. Piscanec, M. Lazzeri, F. Mauri, A. C. Ferrari, and J. Robertson, Phys. Rev. Lett. 93, 185503 (2004).

${ }^{13}$ S. Piscanec, A. C. Ferrari, M. Lazzeri, F. Mauri, and J. Robertson, AIP Conf. Proc. 786, 424 (2005).

${ }^{14}$ S. Piscanec, M. Lazzeri, A. C. Ferrari, F. Mauri, and J. Robertson, Mater. Res. Soc. Symp. Proc. 858E, HH7.4 (2005).

${ }^{15}$ R. Barnett, E. Demler, and E. Kaxiras, Phys. Rev. B 71, 035429 (2005).

${ }^{16}$ A. C. Ferrari, J. C. Meyer, V. Scardaci, C. Casiraghi, M. Lazzeri, F. Mauri, S. Piscanec, D. Jiang, K. S. Novoselov, S. Roth, and A. K. Geim, Phys. Rev. Lett. 97, 187401 (2006).

${ }^{17}$ O. Dubay, G. Kresse, and H. Kuzmany, Phys. Rev. Lett. 88, 235506 (2002).

${ }^{18}$ O. Dubay and G. Kresse, Phys. Rev. B 67, 035401 (2003).

${ }^{19}$ K. P. Bohnen, R. Heid, H. J. Liu, and C. T. Chan, Phys. Rev. Lett. 93, 245501 (2004). 
${ }^{20}$ D. Connetable, G. M. Rignanese, J. C. Charlier, and X. Blase, Phys. Rev. Lett. 94, 015503 (2005).

${ }^{21}$ Y. Huang, M. Okada, K. Tanaka, and T. Yamabe, Phys. Rev. B 53, 5129 (1996).

${ }^{22}$ Y. Huang, M. Okada, K. Tanaka, and T. Yamabe, Solid State Commun. 97, 303 (1996).

${ }^{23}$ A. Sedeki, L. G. Caron, and C. Bourbonnais, Phys. Rev. B 62, 6975 (2000).

${ }^{24}$ M. T. Figge, M. Mostovoy, and J. Knoester, Phys. Rev. Lett. 86, 4572 (2001).

${ }^{25}$ R. A. Jishi, D. Inomata, K. Nakao, M. S. Dresselhaus, and G. Dresselhaus, J. Phys. Soc. Jpn. 63, 2252 (1994).

${ }^{26}$ P. C. Eklund, J. M. Holden, and R. A. Jishi, Carbon 33, 959 (1995).

${ }^{27}$ D. Sanchez-Portal, E. Artacho, J. M. Soler, A. Rubio, and P. Ordejon, Phys. Rev. B 59, 12678 (1999).

${ }^{28}$ J. Yu, R. K. Kalia, and P. Vashishta, Europhys. Lett. 32, 43 (1995).

${ }^{29}$ J. Yu, R. K. Kalia, and P. Vashishta, J. Chem. Phys. 103, 6697 (1995).

${ }^{30}$ M. Menon, E. Richter, and K. R. Subbaswamy, J. Chem. Phys. 104, 5875 (1996).

${ }^{31}$ V. N. Popov and P. Lambin, Phys. Rev. B 73, 085407 (2006).

${ }^{32}$ J. Kurti, G. Kresse, and H. Kuzmany, Phys. Rev. B 58, R8869 (1998).

${ }^{33}$ L. H. Ye, B. G. Liu, D. S. Wang, and H. Rushan, Phys. Rev. B 69, 235409 (2004).

${ }^{34}$ V. N. Popov, V. E. Van Doren, and M. Balkanski, Phys. Rev. B 59, 8355 (1999).

${ }^{35}$ Z. M. Li, V. N. Popov, and Z. K. Tang, Solid State Commun. 130, 657 (2004).

${ }^{36}$ I. Milosevic, E. Dobardzic, and M. Damnjanovic, Phys. Rev. B 72, 085426 (2005).

${ }^{37}$ C. Mapelli, C. Castiglioni, G. Zerbi, and K. Mullen, Phys. Rev. B 60, 12710 (1999).

${ }^{38}$ R. A. Jishi, L. Venkataraman, M. S. Dresselhaus, and G. Dresselhaus, Chem. Phys. Lett. 209, 77 (1993).

${ }^{39}$ J. Maultzsch, S. Reich, C. Thomsen, H. Requardt, and P. Ordejon, Phys. Rev. Lett. 92, 075501 (2004).

${ }^{40}$ V. Zolyomi and J. Kurti, Phys. Rev. B 70, 085403 (2004).

${ }^{41}$ J. Jiang, R. Saito, A. Gruneis, G. Dresselhaus, and M. S. Dresselhaus, Chem. Phys. Lett. 392, 383 (2004).

${ }^{42}$ V. Perebeinos, J. Tersoff, and P. Avouris, Phys. Rev. Lett. 94, 086802 (2005).

${ }^{43}$ G. D. Mahan, Phys. Rev. B 68, 125409 (2003).

${ }^{44}$ S. Baroni, S. de Gironcoli, A. Dal Corso, and P. Giannozzi, Rev. Mod. Phys. 73, 515 (2001).

${ }^{45}$ S. de Gironcoli, Phys. Rev. B 51, 6773 (1995).

${ }^{46}$ R. Saito, M. Fujita, G. Dresselhaus, and M. S. Dresselhaus, Phys. Rev. B 46, 1804 (1992).

${ }^{47}$ J. W. Mintmire, B. I. Dunlap, and C. T. White, Phys. Rev. Lett. 68, 631 (1992).

${ }^{48}$ A. Jorio, M. A. Pimenta, A. G. Souza Filho, G. G. Samsonidze, A. K. Swan, M. S. Unlu, B. B. Goldberg, R. Saito, G. Dresselhaus, and M. S. Dresselhaus, Phys. Rev. Lett. 90, 107403 (2003).

${ }^{49}$ J. Maultzsch, S. Reich, and C. Thomsen, Phys. Rev. B 65, 233402 (2002).

${ }^{50}$ A. Jorio, G. Dresselhaus, M. S. Dresselhaus, M. Souza, M. S. S. Dantas, M. A. Pimenta, A. M. Rao, R. Saito, C. Liu, and H. M.
Cheng, Phys. Rev. Lett. 85, 2617 (2000).

${ }^{51}$ S. Reich, C. Thomsen, and P. Ordejon, Phys. Rev. B 64, 195416 (2001).

${ }^{52}$ R. Saito, A. Jorio, J. H. Hafner, C. M. Lieber, M. Hunter, T. McClure, G. Dresselhaus, and M. S. Dresselhaus, Phys. Rev. B 64, 085312 (2001).

${ }^{53}$ W. Kohn, Phys. Rev. Lett. 2, 393 (1959).

${ }^{54}$ N. W. Ashcroft and N. D. Mermin, Solid State Physics (Saunders College, New York, London, 1976).

${ }^{55}$ R. E. Peierls, Quantum Theory of Solids (Clarendon Press, Oxford, 1955).

${ }^{56}$ L. X. Benedict, V. H. Crespi, S. G. Louie, and M. L. Cohen, Phys. Rev. B 52, 14935 (1995).

${ }^{57}$ Z. K. Tang, L. Y. Zhang, N. Wang, X. X. Zhang, G. H. Wen, G. D. Li, J. N. Wang, C. T. Chan, and P. Sheng, Science 292, 2462 (2001).

${ }^{58}$ F. Mauri (unpublished).

${ }^{59}$ N. D. Mermin, Phys. Rev. 137, A1441 (1965).

${ }^{60}$ Note that in Ref. 12 the dynamical matrix projected on the phonon normal coordinate was indicated as $D_{\mathbf{q}}$. In this paper, and in Ref. $10, D_{(\mathbf{k}+\mathbf{q}) \mathbf{i}, \mathbf{k j}}$ represents the electron-phonon coupling matrix element. Thus, to avoid ambiguities, in this work we replace the symbol $D_{\mathbf{q}}$ with $\Theta_{\mathbf{q}}$.

${ }^{61}$ P. B. Allen, in Dynamical Properties of Solids, edited by G. K. Horton and A. A. Maradudin (North-Holland, Amsterdam, 1980), Vol. 3, pp. 95-196.

${ }^{62}$ S. Piscanec, M. Lazzeri, F. Mauri, J. Robertson, and A. C. Ferrari, Abstract E 5 03, e-MRS 2006 Spring Meeting abstract book, 2006.

${ }^{63}$ M. Born and R. Oppenheimer, Ann. Phys. 84, 457 (1927).

${ }^{64}$ L. J. Sham, in Dynamical Properties of Solids, edited by G. K. Horton and A. A. Maradudin (North-Holland, Amsterdam, 1974), Vol. 1, p. 318.

${ }^{65}$ M. T. Yin and M. L. Cohen, Phys. Rev. B 26, 3259 (1982).

${ }^{66}$ R. M. Martin, Electronic Structure, Basic Theory and Practical Methods (Cambridge University Press, Cambridge, UK, 2004).

${ }^{67}$ Note that the $3 / 2$ prefactor reported for this equation in Ref. 10 is a typographical error.

${ }^{68} \mathrm{http}: / /$ www.pwscf.org/guide/3.0/html-single/manual.html

${ }^{69} \mathrm{http}: / / \mathrm{www} . c p m d . o r g / c p m d-o n-l i n e-m a n u a l . h t m l$

${ }^{70}$ M. Lazzeri, S. Piscanec, F. Mauri, A. C. Ferrari, and J. Robertson, Phys. Rev. B 73, 155426 (2006).

${ }^{71}$ S. Koswatta, S. Hasan, M. S. Lundstrom, M. P. Anantram, and D. E. Nikonov, cond-mat/0511723 (2005).

${ }^{72}$ M. Abramowitz and I. A. Stegun, Handbook of Mathematical Functions (National Brueau of Standards, Dover, New York, 1972).

${ }^{73}$ R. Barnett, E. Demler, and E. Kaxiras, Solid State Commun. 135, 335 (2005).

${ }^{74}$ V. N. Popov, New J. Phys. 6, 17 (2004).

${ }^{75}$ M. Methfessel and A. T. Paxton, Phys. Rev. B 40, 3616 (1989); calculations on metals are often performed by smearing the electron occupation using various distribution functions. This is done since it smoothens the discontinuities at the Fermi energy, and thus greatly increases the speed of calculations. The smearing parameter $\sigma$ in the distribution function is sometimes interpreted as a fictitious electronic temperature $T_{f}$.

${ }^{76}$ J. Maultzsch, S. Reich, U. Schlecht, and C. Thomsen, Phys. Rev. Lett. 91, 087402 (2003).

${ }^{77}$ S. Piscanec, V. Scardaci, M. Lazzeri, R. Krupke, F. Mauri, and A. C. Ferrari (unpublished). 
${ }^{78}$ M. Lazzeri and F. Mauri, Phys. Rev. Lett. 97, 266407 (2006).

${ }^{79}$ S. Pisana, M. Lazzeri, C. Casirgahi, K. S. Novoselov, A. K. Geim, A. C. Ferrari, and F. Mauri (unpublished).

${ }^{80}$ M. S. Dresselhaus, G. Dresselhaus, R. Saito, and A. Jorio, Phys. Lett., C 409C, 47 (2005).

${ }^{81}$ S. Reich and C. Thomsen, Philos. Trans. R. Soc. London, Ser. A 362, 2271 (2004).

${ }^{82}$ A. Jorio, R. Saito, G. Dresselhaus, and M. S. Dresselhaus, Philos. Trans. R. Soc. London, Ser. A 362, 2311 (2004).

${ }^{83}$ F. Tuinstra and J. Koenig, J. Chem. Phys. 53, 1126 (1970).

${ }^{84}$ A. C. Ferrari and J. Robertson, Phys. Rev. B 61, 14095 (2000).

${ }^{85}$ A. C. Ferrari and J. Robertson, Phys. Rev. B 64, 075414 (2001).

${ }^{86}$ A. Jorio, A. G. Souza, G. Dresselhaus, M. S. Dresselhaus, A. K. Swan, M. S. Unlu, B. B. Goldberg, M. A. Pimenta, J. H. Hafner, C. M. Lieber et al., Phys. Rev. B 65, 155412 (2002).

${ }^{87}$ M. A. Pimenta, A. Marucci, S. A. Empedocles, M. G. Bawendi, E. B. Hanlon, A. M. Rao, P. C. Eklund, R. E. Smalley, G. Dresselhaus, and M. S. Dresselhaus, Phys. Rev. B 58, R16016 (1998).

${ }^{88}$ H. Kataura, Y. Kumazawa, Y. Maniwa, I. Umezu, S. Suzuki, Y. Ohtsuka, and Y. Achiba, Synth. Met. 103, 2555 (1999).

${ }^{89}$ P. M. Rafailov and C. Thomsen, J. Optoelectron. Adv. Mater. 7, 461 (2005).

${ }^{90}$ S. D. M. Brown, A. Jorio, P. Corio, M. S. Dresselhaus, G. Dresselhaus, R. Saito, and K. Kneipp, Phys. Rev. B 63, 155414
(2001).

${ }^{91}$ C. Y. Jiang, K. Kempa, J. L. Zhao, U. Schlecht, U. Kolb, T. Basche, M. Burghard, and A. Mews, Phys. Rev. B 66, 161404(R) (2002).

${ }^{92}$ K. Kempa, Phys. Rev. B 66, 195406 (2002).

${ }^{93}$ J. Maultzsch, S. Reich, U. Schlecht, and C. Thomsen, Phys. Rev. Lett. 91, 087402 (2003).

${ }^{94}$ H. Telg, J. Maultzsch, S. Reich, F. Hennrich, and C. Thomsen, Phys. Rev. Lett. 93, 177401 (2004).

${ }^{95}$ M. Oron-Carl, F. Hennrich, M. M. Kappes, H. V. Lohneysen, and R. Krupke, Nano Lett. 5, 1761 (2005).

${ }^{96}$ S. K. Doorn, M. J. O'Connell, L. X. Zheng, Y. T. T. Zhu, S. M. Huang, and J. Liu, Phys. Rev. Lett. 94, 016802 (2005).

${ }^{97}$ M. Paillet, P. Poncharal, A. Zahab, J. L. Sauvajol, J. C. Meyer, and S. Roth, Phys. Rev. Lett. 94, 237401 (2005).

${ }^{98}$ S. M. Bose, S. Gayen, and S. N. Behera, Phys. Rev. B 72, 153402 (2005).

${ }^{99}$ J. C. Meyer, M. Paillet, T. Michel, A. Moreac, A. Neumann, G. S. Duesberg, S. Roth, and J. L. Sauvajol, Phys. Rev. Lett. 95, 217401 (2005).

${ }^{100}$ E. B. Barros, A. Jorio, G. G. Samsonidze, R. B. Capaz, A. G. Souza Filho, J. Mendes Filho, G. Dresselhaus, and M. S. Dresselhaus, Phys. Rep. 431, 261 (2006).

${ }^{101}$ C. Thomsen and S. Reich, Phys. Rev. Lett. 85, 5214 (2000). 